Research Paper

\title{
Forsythoside A Mitigates Alzheimer's-like Pathology by Inhibiting Ferroptosis-mediated Neuroinflammation via Nrf2/GPX4 Axis Activation
}

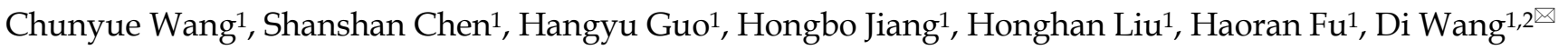 \\ 1. School of Life Sciences, Jilin University, Changchun, 130012, China. \\ 2. Engineering Research Center of Chinese Ministry of Education for Edible and Medicinal Fungi, Jilin Agricultural University, Changchun, 130118, China. \\ $\square$ Corresponding author: Di Wang, Ph.D., Professor, School of Life Sciences, Jilin University, Engineering Research Center of Chinese Ministry of Education for \\ Edible and Medicinal Fungi, Jilin Agricultural University. E-mail: jluwangdi@jlu.edu.cn.
}

(C) The author(s). This is an open access article distributed under the terms of the Creative Commons Attribution License (https://creativecommons.org/licenses/by/4.0/). See http://ivyspring.com/terms for full terms and conditions.

Received: 2021.12.04; Accepted: 2022.02.03; Published: 2022.02.28

\begin{abstract}
Ferroptosis and neuroinflammation play crucial roles in Alzheimer's disease (AD) pathophysiology. Forsythoside A (FA), the main constituent of Forsythia suspensa (Thunb.) Vahl., possesses anti-inflammatory, antibacterial, antioxidant, and neuroprotective properties. The present study aimed to investigate the potential role of FA in AD neuropathology using male APP/PS1 double transgenic AD mice, $A \beta_{1-42-e x p o s e d ~} N 2$ a cells, erastin-stimulated $H T 22$ cells, and LPS-induced BV2 cells. FA treatment

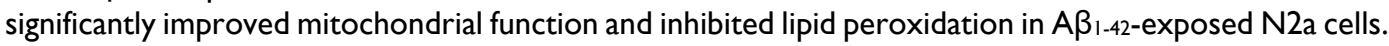
In LPS-stimulated BV2 cells, FA treatment decreased the formation of the pro-inflammatory factors IL-6, IL-1 $\beta$, and NO. In male APP/PSI mice, FA treatment ameliorated memory and cognitive impairments and suppressed $A \beta$ deposition and $P$-tau levels in the brain. Analyses using proteomics, immunohistochemistry, ELISA, and western blot revealed that FA treatment significantly augmented dopaminergic signaling, inhibited iron deposition and lipid peroxidation, prevented the activation of $\mathrm{IKK} / \mathrm{IKB} / \mathrm{NF}-\mathrm{KB}$ signaling, reduced the secretion of pro-inflammatory factors, and promoted the production of anti-inflammatory factors in the brain. FA treatment exerted anti-ferroptosis and anti-neuroinflammatory effects in erastin-stimulated HT22 cells, and the Nrf2/GPX4 axis played a key role in these effects. Collectively, these results demonstrate the protective effects of FA and highlight its therapeutic potential as a drug component for AD treatment.
\end{abstract}

Key words: Alzheimer's disease, forsythoside A, neuroprotection, neuroinflammation, ferroptosis, Nrf2/GPX4 axis

\section{Introduction}

Alzheimer's disease (AD) is the most prevalent neurodegenerative disorder causing dementia [1]. The pathophysiological processes underscoring $\mathrm{AD}$ are thought to begin 20 years or more prior to the onset of cognitive symptoms [2], which negatively impact patients' health and place a substantial economic burden on individuals, families, and society [3]. In 2019, the oligosaccharide GV-971 was approved for the treatment of mild and moderate AD in China and was assessed in an international phase III clinical trial in April 2020 [4]. In June 2021, the U.S. Food and Drug Administration approved aducanumab, co-developed by Biogen and Eisai, for the treatment of AD [5]. Despite these encouraging findings, the pathological mechanisms underlying AD remain unclear, which precludes the development of more effective treatments [6].

Iron deposits are observed in plaques, microglia, and tangle-bearing neurons in the AD brain [7]. Iron is a key component of ferroptosis, which is defined as the accumulation of lethal lipid substances produced by lipid peroxidation. Ferroptosis has emerged as a major process in $\mathrm{AD}$ pathophysiology [8] and has become a target for AD treatment. Previous studies have demonstrated that almost all genes related to ferroptosis are regulated by nuclear factor erythroid 2-related factor 2 (Nrf2) transcription, including, but not limited to, nicotinamide adenine 
dinucleotide phosphate, glutathione peroxidase 4 (GPX4), and iron-regulated genes [8, 9]. As a member of the selenoprotein glutathione peroxidase family, GPX4 has the unique function of reducing hydroperoxide in membrane lipids [10]. This property of reducing phospholipid hydroperoxide, thus inhibiting lipoxygenase-mediated lipid peroxidation, underscores its vital role in preventing ferroptosis [8]. Nevertheless, the role of Nrf2/GPX4 in AD remains poorly understood.

In addition to ferroptosis, brain tissue is particularly vulnerable to neuroinflammation, which can aggravate amyloid- $\beta$ (A $\beta$ ) deposition and tau phosphorylation [11]. Ferroptosis is associated with neuroinflammation, suggesting that iron retention can trigger microglial activation and interleukin (IL)- $1 \beta$ production in AD [7]. Additionally, the Nrf2 pathway inhibits reactive oxygen species (ROS)-related nuclear factor- $\mathrm{K} B(\mathrm{NF}-\mathrm{k} B)$ activation by preventing the degradation of inhibitor of NF-kB (IкB)- $\alpha$ [12]. As a downstream target of Nrf2, GPX4 prevents tumor necrosis factor (TNF)-mediated activation of NF- $\mathrm{kB}$. Correspondingly, neuroinflammation is suppressed via inhibition of ferroptosis [13]. Although ferroptosis inhibitors are considered as key regulators of neuroinflammation, the role of Nrf2/GPX4 in the regulation of the NF- $\mathrm{kB}$ signaling pathway in AD has yet to be elucidated.

Forsythoside A (FA) (3,4-dihydroxy- $\beta$ phenethyl-O- $\alpha-L-r h a m n o p y r a n o s y l-(1 \rightarrow 6)-4-O-c a f f e o$ yl- $\beta$-D-glucopyranoside) (Fig. 1A), also referred to as forsythiaside, is the main component of Forsythia suspensa (Thunb.) Vahl (F. suspensa or Lianqiao in Chinese), which was first recorded in Shen Nong's Herbology, the earliest extant treatise in Chinese pharmacological literature [14]. FA possesses various beneficial pharmacological characteristics, including anti-inflammatory, anti-oxidative, and neuroprotective properties [15]. Oral administration of FA was reported to decrease the number of microglia and astrocytes in transient cerebral global ischemia in gerbils [16]. Further, FA treatment attenuated neuroinflammation and apoptosis caused by $A \beta$ in hippocampal slices [17], improved memory and learning abilities in senescence-accelerated mouseprone 8 mice [15], and reduced $A \beta_{25-35}$-induced apoptosis in PC12 cells [18]. However, the effects of FA treatment on ferroptosis in AD have not been reported to date.

In this study, we explored the effects of FA treatment on AD neuropathology in male APP/PS1 double transgenic $\mathrm{AD}$ mice, $\mathrm{A} \beta_{1-42}$-exposed N2a cells, erastin-stimulated HT22 cells, and lipopolysaccharide (LPS)-induced BV2 cells in order to identify its mechanism of action.

\section{Materials and methods}

\section{Animals and ethical statement}

All animal experiments were approved by the Animal Ethics Committee of Jilin University (permit No. SY201905013) and were conducted in compliance with the ARRIVE guidelines. Eight-month-old B6C3-Tg (APPswePSEN1dE9)/Nju double transgenic male mice (APP/PS1) (genotype: (Appswe) T, (Psen1) $\mathrm{T}$ ) and age-matched wild-type (WT) (genotype: (Appswe) W, (Psen1) W) male mice were purchased from Nanjing Biomedical Research Institute of Nanjing University. All mice were individually housed at $24{ }^{\circ} \mathrm{C}$ with food and drinking water available ad libitum. After 1 week of adaption in the new environment, WT mice received oral administration of normal saline $(10 \mathrm{~mL} / \mathrm{kg})$ and were designated as the control group $(n=12)$. APP/PS1 mice were randomly divided into two groups: the model group $(n=12)$ received oral administration of normal saline $(10 \mathrm{~mL} / \mathrm{kg})$ and the agent-treated group $(n=12)$ received oral treatment with $30 \mathrm{mg} / \mathrm{kg}$ FA (L-012-171216, 98.83\% purity, Chengdu Herbpurify Co., Ltd., Chengdu, China) beginning on day 8. After 30-day treatment, behavioral experiments were serially performed. The entire treatment protocol lasted for 42 days. Blood samples were collected from the caudal vein. After euthanasia via $\mathrm{CO}_{2}$ inhalation, organs including the brain, liver, spleen, and kidney were collected for further analysis (Fig. 2A).

\section{Behavioral tests}

\section{Y-maze test}

Working memory was evaluated using the Y-maze test. The Y-maze comprised three arms (dimensions of each arm: $30 \mathrm{~cm} \times 6 \mathrm{~cm} \times 30 \mathrm{~cm}$ ), including one start arm and two objective arms. After 3-day training, the mice were fasted for $12 \mathrm{~h}$ prior to the formal test. On day 41 , the mice were individually placed at the end of the start arm, and food was placed in one of the two objective arms. The movement trajectory of each mouse and time spent searching for food were recorded with a camera and analyzed using Any-maze software (Stoelting Co., Chicago, IL, USA) under the condition of researchers blinded to treatment groups.

\section{Morris water maze (MWM) test}

Spatial memory and navigation ability were evaluated using the MWM test on day 43. Both training and formal testing were performed using an MT-200 water labyrinth video-tracking analysis system (MT-200, TECHMAN Software Co., Ltd., Chengdu, China). The cistern was divided into four quadrants. Titanium dioxide was added to the cistern 
to make the water turbid. During training, the mice were placed in the water to reach the platform within $60 \mathrm{~s}$. After finding the platform, the mice stayed on the platform for $20 \mathrm{~s}$. The mice that did not find the platform within $60 \mathrm{~s}$ were manually led to the platform and stayed there for $20 \mathrm{~s}$. After 3 days of training, the navigation test and probe trial were performed under blinded conditions on days 46 and 47, respectively. As reported in our previous study [19], the mice were placed into the cistern from the opposite side of the platform, and the time it took for mice to find the platform and/or the time they passed through the effective area were recorded.

\section{Pathology assessments}

Immunohistochemistry assays were conducted as reported previously [20]. Briefly, 4\% paraformaldehyde-fixed brain slides were deparaffinized, and then antigen retrieval was performed with a citric acid antigen retrieval buffer (pH 6.0) (G1202, servicebio technology CO., LTD, Wuhan, China) in a microwave oven. After incubating with primary antibodies (Table S1) at $4{ }^{\circ} \mathrm{C}$ overnight, the sections were incubated with biotin-conjugated AffiniPure goat anti-rabbit IgG $(\mathrm{H}+\mathrm{L})$ (Table S1). Images were obtained using an optical microscope (BX51, Olympus, Tokyo, Japan).

\section{Label-free quantification proteomics}

Label-free quantification proteomics was performed as previously described [19]. Briefly, 100 $\mathrm{mg}$ of mouse hippocampal tissue was collected with $1,000 \mu \mathrm{L}$ ice-cold radio immunoprecipitation assay (RIPA) (20-188, Sigma-Aldrich, St. Louis, MO, USA) containing $1 \%$ proteolytic protease and phosphatase inhibitor cocktail (P002, New Cell \& Molecular Biotech Co., Ltd., Suzhou, China), which were added for tissue homogenization at $4{ }^{\circ} \mathrm{C}$. After centrifugation at $4{ }^{\circ} \mathrm{C}$ for $15 \mathrm{~min}$, proteins were precipitated using acetone. The samples were then resuspended and mixed with trypsin, incubated overnight at $37^{\circ} \mathrm{C}$, and then desalted after the removal of sodium deoxycholate. A volume of $2 \mu \mathrm{L}$ of polypeptide was analyzed using nano ultra-performance liquid chromatography (EASY-nLC1200) coupled with Q-Exactive mass spectrometry (Thermo Finnigan). MaxQuant (Version 1.5.6.0) was used to analyze the raw MS files. The protein sequence database was obtained from UniProt (Uniprot_mouse_2016_09). Significantly differentially expressed proteins were defined as proteins with a content ratio between the two groups (WT group/APP/PS1 group or FA-treated APP/PS1 group/APP/PS1 group) of $>1.5$ or $<0.66$. These differentially expressed proteins were subjected to cluster analysis, protein interaction analysis, gene ontology (GO) analysis, and Kyoto Encyclopedia of Genes and Genomes (KEGG) analysis.

\section{Cellular experiments}

\section{Cell cultures}

HT22 cells derived from a mouse hippocampal neuronal cell line (CL-0595, Procell Life Science \& Technology Co., Ltd., Wuhan, China) were cultured in Dulbecco's modified Eagle's medium (C11995500BT, Thermo Fisher Biochemical Products Co., Ltd., Waltham, MA, USA) containing $10 \%$ fetal bovine serum (164210, Procell Life Science \& Technology Co., Ltd.), and 1\% penicillin-streptomycin solution (15140, Thermo Fisher Biochemical Products Co., Ltd.). Mouse neuroblastoma N2a cells (CX0020, Boster Biological Technology Co., Ltd., Wuhan, China) and BV2 microglial cells (CL-0493, Procell Life Science \& Technology Co., Ltd.) were maintained in minimum essential medium (PM150410, Procell Life Science \& Technology Co., Ltd.) supplemented with $10 \%$ fetal bovine serum and $1 \%$ penicillin-streptomycin solution. Cells were cultured in a humidified atmosphere with $5 \% \mathrm{CO}_{2}$ at $37^{\circ} \mathrm{C}$.

\section{Cell viability assay}

HT22 cells were seeded in a 96-well plate $\left(5 \times 10^{3}\right.$ cells per well) and pretreated with FA $(40 \mu \mathrm{M}$ and 80 $\mu \mathrm{M})$ for $3 \mathrm{~h}$, followed by $24-\mathrm{h}$ co-exposure to $10 \mu \mathrm{M}$ of erastin (HY-15763, MedChemExpress, Shanghai, China). N2a cells $\left(5 \times 10^{3}\right.$ cells per well $)$ were seeded in a 96-well plate and pretreated with FA $(40 \mu \mathrm{M}$ and $80 \mu \mathrm{M}$ ) for $3 \mathrm{~h}$, followed by 24-h co-exposure to $10 \mu \mathrm{M}$ of A $\beta$ oligomers (052487, GL Biochem Ltd., Shanghai, China). Cell viability was measured using 3-(4,5dimethylthiazolyl-2-yl)-2,5-diphenyl tetrazolium bromide (MTT) (S19063, Shanghai Yuanye Bio-Technology Co., Ltd., Shanghai, China) at 490 nm, as described in our previous study [20].

\section{Mitochondrial membrane potential (MMP) detection}

MMP was evaluated using 5,5',6,6'-Tetrachloro1,1',3,3'-tetraethyl-imidacarbocyanine iodide (JC-1) staining (C2006, Beyotime, Shanghai, China) according to the manufacturer's instructions. N2a cells $\left(2 \times 10^{5}\right.$ cells per well) were seeded in a 6-well plate and incubated overnight. Cells were pretreated with FA $(40 \mu \mathrm{M}$ and $80 \mu \mathrm{M})$ for $3 \mathrm{~h}$ followed by stimulation with $10 \mu \mathrm{M}$ of $\mathrm{A} \beta$ oligomers. After 24 -h co-incubation, cells were incubated with $500 \mu \mathrm{L}$ of JC- 1 at $37{ }^{\circ} \mathrm{C}$ for $30 \mathrm{~min}$ in the dark. Cells were washed in phosphate buffer solution (PBS), and fluorescence images were subsequently obtained using a fluorescence microscope (TH4-200, Olympus 
Corporation, Japan).

\section{Glutathione (GSH) assay}

HT22 cells were pretreated with $40 \mu \mathrm{M}$ and 80 $\mu \mathrm{M}$ of FA for $3 \mathrm{~h}$, followed by co-incubation with 10 $\mu \mathrm{M}$ of erastin for another $24 \mathrm{~h}$. GSH content in HT22 cells was evaluated according to the manufacturer's instructions (S0053, Beyotime, Shanghai, China).

\section{Lipid ROS detection}

HT22 cells $\left(2 \times 10^{5}\right.$ cells per well $)$ were seeded in a 6-well plate and pretreated with FA $(40 \mu \mathrm{M}$ and 80 $\mu \mathrm{M})$ for $3 \mathrm{~h}$, followed by 24 -h co-exposure to $10 \mu \mathrm{M}$ of erastin. Cells were incubated with BODIPY 581/591 C11 (D3861, Thermo Fisher Biochemical Products Co., Ltd., Waltham, MA, USA) at a final concentration of $10 \mu \mathrm{M}$ for $30 \mathrm{~min}$ in the dark. After three washes in PBS, fluorescence images were obtained using a fluorescence microscope (TH4-200, Olympus Corporation, Japan).

\section{Griess assay}

The amount of nitric oxide (NO) produced by BV2 cells is positively related to nitrite release, which is considered an indicator of NO generation. BV2 cells were pretreated with FA $(40 \mu \mathrm{M}$ and $80 \mu \mathrm{M})$ for $3 \mathrm{~h}$, followed by 24-h co-exposure to $1 \mu \mathrm{g} / \mathrm{mL}$ of LPS (DH183-1, Beijing Dingguo Changsheng Biotechnology Co., Ltd., Beijing, China). A volume of $50 \mu \mathrm{L}$ of supernatant was collected and added to a fresh 96-well plate containing an equivalent volume of Griess reagent I and Griess reagent II (S0021, Beyotime, Shanghai, China). The absorbance was measured at $540 \mathrm{~nm}$ using a microwave reader (HBS-1096A, Detie, Nanjing, China).

\section{Transmission electron microscopy (TEM)}

As described in our previous study [19]. HT22 cells were pretreated with FA $(40 \mu \mathrm{M}$ and $80 \mu \mathrm{M})$ for 3 $\mathrm{h}$, followed by co-incubation with $10 \mu \mathrm{M}$ of erastin for $24 \mathrm{~h}$. After fixation with $2.5 \%$ glutaraldehyde at $4{ }^{\circ} \mathrm{C}$ for $4 \mathrm{~h}$, the cells were fixed with $1 \%$ osmic acid- $0.1 \mathrm{M}$ PBS (pH 7.4) for $2 \mathrm{~h}$ at $25^{\circ} \mathrm{C}$, dehydrated in an ethanol gradient, permeabilized, and polymerized at $60^{\circ} \mathrm{C}$ for $48 \mathrm{~h}$. Ultrathin sections of $60-80 \mathrm{~nm}$ were prepared and stained with a saturated aqueous solution of $2 \%$ uranyl acetate and lead citrate. The ultrastructure of HT22 cells was observed using TEM (TECNAI G2 20 TWIN, FEI, Hillsboro, OR, USA).

\section{RNA interference (RNAi) of GPX4 and Nrf2}

HT22 cells were transfected with small interfering RNA (siRNA) targeting GPX4 (50 nM) (Q1208, RiboBio, Guangzhou, China) and Nrf2 (50 nM) (U0805, RiboBio, Guangzhou, China) to knock down the expression of GPX4 and Nrf2, respectively.
Negative control (NC) siRNA (50 nM) (S1012, RiboBio, Guangzhou, China) served as the control group. After $24 \mathrm{~h}$ of transfection, HT22 cells were treated with FA or erastin and then used for subsequent experiments. All transfections were performed using LipoRNAi transfection reagent (C0535, Beyotime, Shanghai, China).

\section{Malondialdehyde (MDA) assay}

Normal or GPX4/Nrf2 siRNA-transfected HT22 cells were pretreated with FA $(40 \mu \mathrm{M}$ and $80 \mu \mathrm{M})$ for 3 $\mathrm{h}$, followed by co-incubation with $10 \mu \mathrm{M}$ of erastin for $24 \mathrm{~h}$. Normal N2a cells were pretreated with FA (40 $\mu \mathrm{M}$ and $80 \mu \mathrm{M}$ ) for $3 \mathrm{~h}$, followed by co-incubation with $10 \mu \mathrm{M}$ of $\mathrm{A} \beta$ oligomers for $24 \mathrm{~h}$. All treated cells were collected, and the protein concentration was determined using a BCA Protein Assay Kit (23225, Thermo Fisher Scientific, Waltham, MA, USA) according to the manufacturer's instructions. MDA production was analyzed using a cell MDA assay kit (A003-4-1, Nanjing Jiancheng Bioengineering Institute, Nanjing, China) according to the manufacturer's instructions.

\section{Enzyme-linked immunosorbent assay (ELISA)}

Brain samples were collected from APP/PS1 mice and were lysed using PBS buffer. Protein concentration was determined using a BCA Protein Assay Kit. The levels of transforming growth factor- $\beta$ (TGF- $\beta$ ) (RK00057), monocyte chemoattractant protein-1 (MCP-1) (RK00381), IL-1 $\beta$ (RK00006), IL-6 (RK00008), and TNF-a (RK00027) in brains were analyzed using a commercial kit (ABclonal, Wuhan, China) according to the manufacturer's instructions. BV2 cells were pretreated with FA $(40 \mu \mathrm{M}$ and $80 \mu \mathrm{M})$ for $3 \mathrm{~h}$, followed by co-incubation with $1 \mu \mathrm{g} / \mathrm{mL}$ of LPS for $24 \mathrm{~h}$. The levels of IL-1 $\beta$ (EK0394) and IL-6 (EK0411) in the collected culture medium were analyzed using a commercial kit (Boster, Wuhan, China) according to the manufacturer's instructions.

\section{Western blot}

Normal or GPX4/Nrf2 siRNA-transfected HT22 cells were pretreated with FA $(40 \mu \mathrm{M}$ and $80 \mu \mathrm{M})$ for 3 $\mathrm{h}$, followed by co-incubation with $10 \mu \mathrm{M}$ of erastin for $24 \mathrm{~h}$. Brain samples were collected from APP/PS1 mice, and the collected cells were lysed using ice-cold RIPA containing $1 \%$ lytic protease and phosphatase inhibitor cocktail. Protein concentration was determined using a BCA Protein Assay Kit. Protein samples were separated by sodium dodecyl sulfatepolyacrylamide gel electrophoresis and transferred to polyvinylidene fluoride membranes $(0.45 \mu \mathrm{m})$ (10600023, Cytiva, Breisgau, Germany). Membranes were blocked with a highly efficient commercial blocking solution (GF1815, Genefist, Culham Science 
Centre, Oxfordshire, UK) for $15 \mathrm{~min}$, followed by overnight incubation with primary antibodies (Table S1) at $4{ }^{\circ} \mathrm{C}$ and 2-h incubation with horseradish peroxidase-conjugated secondary antibodies (Table S1) at $25^{\circ} \mathrm{C}$. The protein bands were detected using an ultra-sensitive electrochemiluminescence kit (P2300,
New Cell \& Molecular Biotech Co., Ltd., Suzhou, China) with an imaging system (Tanon 5200, Tanon Science \& Technology Co., Ltd., Shanghai, China) and analyzed using ImageJ 6.0 software (National Institutes of Health, Bethesda, Maryland, USA).
A<smiles>C[C@@H]1O[C@H](OC[C@H]2O[C@H](OCCc3ccc(O)c(O)c3)[C@@H](O)[C@H](O)[C@H]2OC(=O)/C=C/c2ccc(O)c(O)c2)[C@H](O)[C@@H](O)[C@@H]1O</smiles>
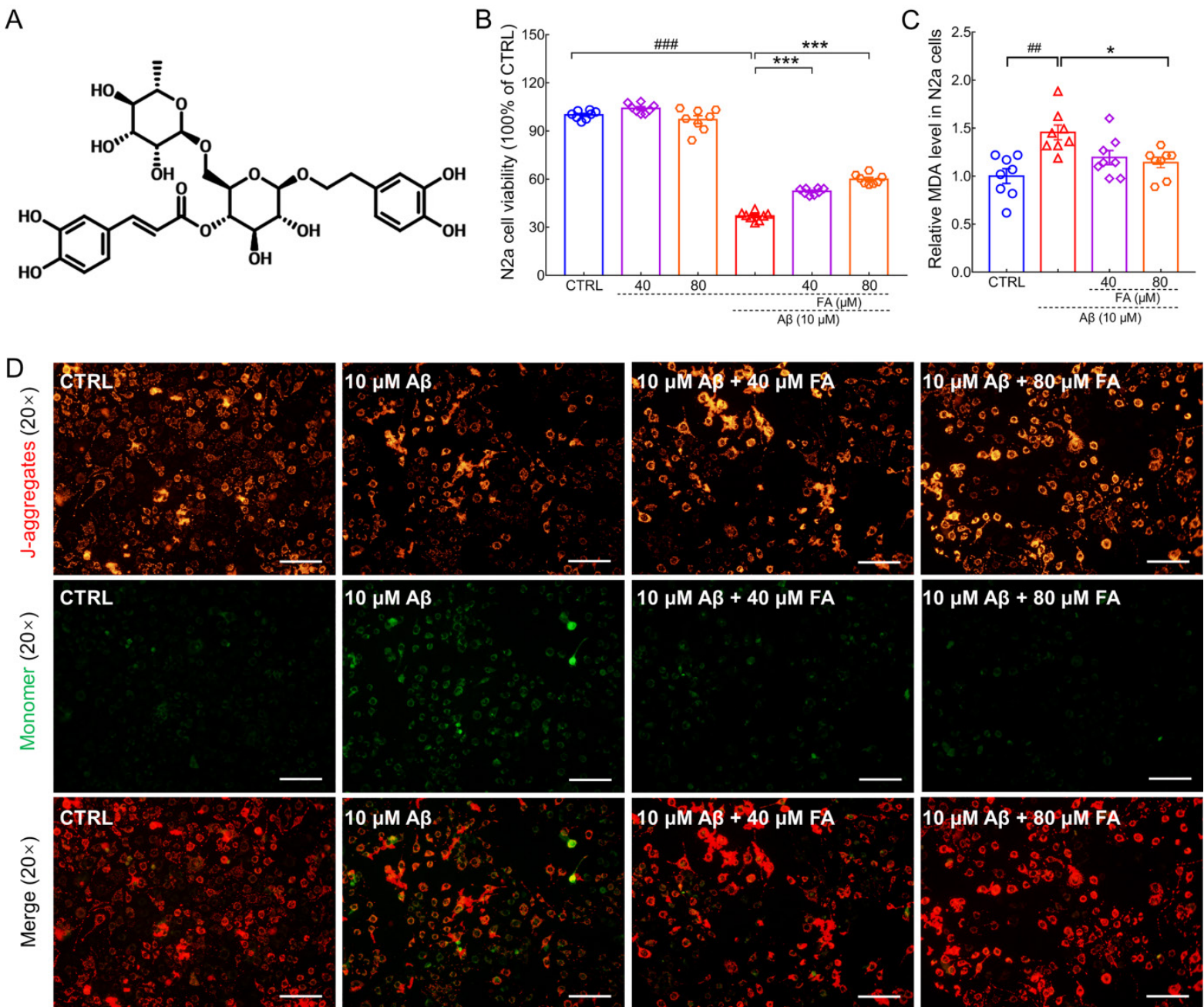

E
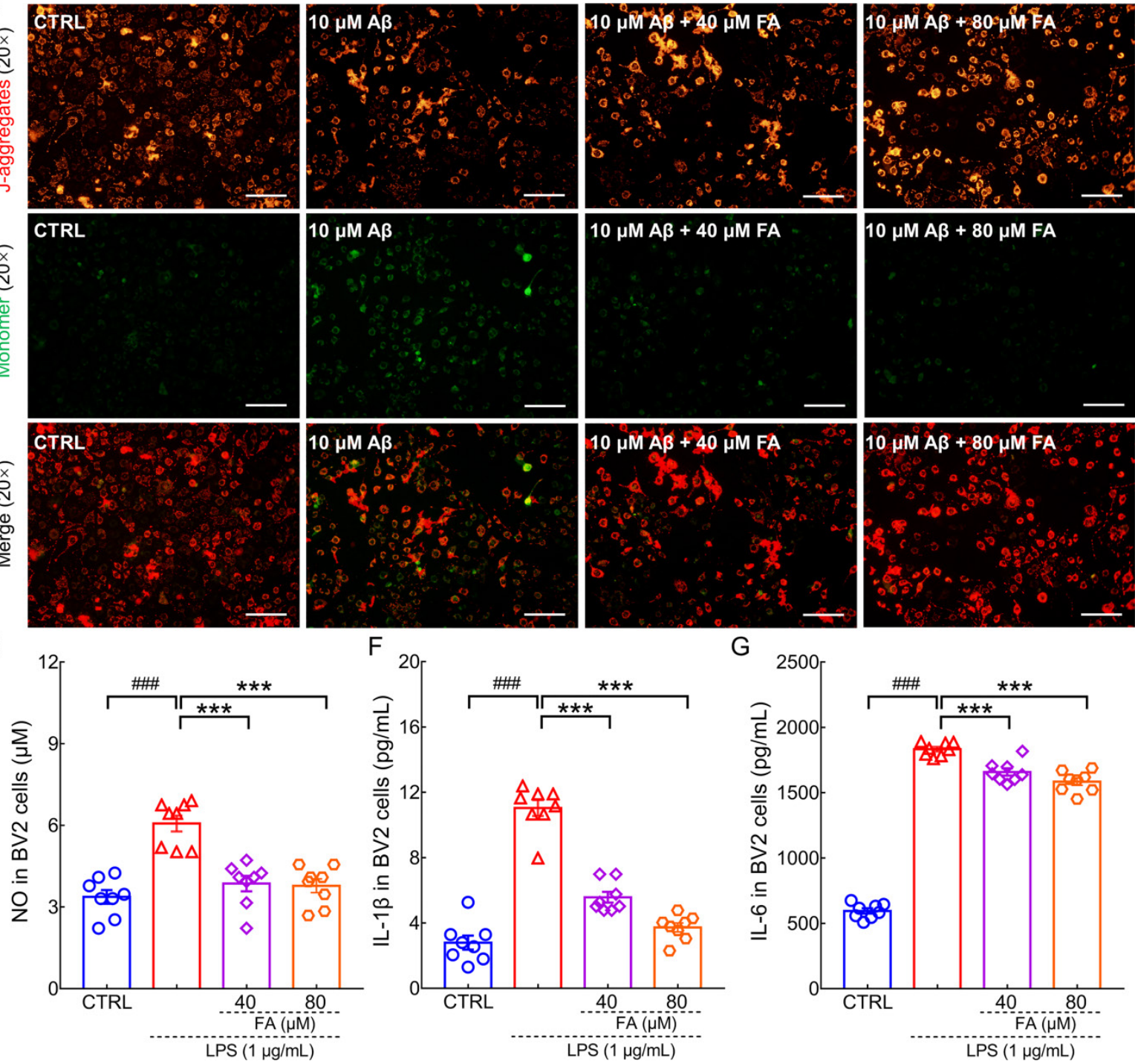

Figure 1. FA treatment protects $\mathbf{N 2 a}$ cells and BV2 cells against $\mathbf{A} \boldsymbol{\beta}_{1-42}$ and LPS toxicity. (A) Chemical structure of FA (CAS: 79916-77-1). (B) FA treatment enhanced cell viability in $A \beta_{1-42}$-exposed $N 2$ a cells without influencing cell viability alone $(n=8)$. (C) FA treatment downregulated MDA levels in A 1 I-42-exposed N2a cells $(n=$ 8). (D) FA treatment prevented the dissipation of MMP in $A \beta_{1-42}$-exposed N2a cells $(n=3)$. Scale bar: $100 \mu \mathrm{m}$. Red and green fluorescence signals represent J-aggregates and monomers, respectively. FA treatment decreased the concentration of $(\mathbf{E}) \mathrm{NO}$, (F) IL-1 $\beta$, and $(\mathbf{G}) \mathrm{IL}-6$ in LPS-exposed BV2 cells $(n=8)$. The data are presented as mean \pm S.E.M.

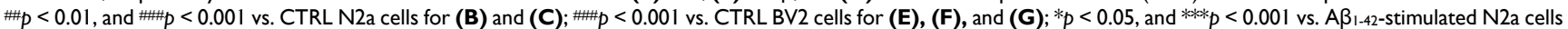
for (B) and (C); ***p $<0.001$ vs. LPS-exposed BV2 cells for (E), (F), and (G). 


\section{Statistical analysis}

All data are presented as mean \pm S.E.M. BONC DSS Statistics 25 software (Business-intelligence of Oriental Nations Corporation Ltd., Beijing, China) was used for statistical analysis, and GraphPad Prism 9 software (GraphPad Software Inc., San Diego, CA, USA) was used for generating graphs. A one-way analysis of variance (ANOVA) followed by Tukey's post hoc test was performed. Statistical significance was set at $p<0.05$.

\section{Results}

\section{FA treatment protected cells against $A \beta_{1-42}$ and LPS-induced injury}

$A \beta$ is thought to promote neuronal death by damaging mitochondria, aggravating neuroinflammation and oxidative stress [21]. Inflammation of the central nervous system is closely associated with neurodegenerative diseases. Microglia own an important position and number in neuronal cell populations in the brain region and are activated during neuroinflammation [22]. In this study, the classic preclinical AD models of $A \beta_{1-42}$-induced $\mathrm{N} 2 \mathrm{a}$ cells and LPS-induced BV2 microglia were used to explore the neuroprotective effects of FA. FA treatment dose-dependently improved cell viability ( $p$ $<0.001$ ) (Fig. 1B), decreased MDA levels $(p<0.05)$ (Fig. 1C), and prevented the dissipation of MMP $(p<$ 0.01 ) (Fig. $1 D$ and Fig. S1) in $A \beta_{1-42}$-exposed N2a cells, suggesting that $F A$ protects against $A \beta_{1-42}$ toxicity. LPS has been reported to induce inflammation in microglia [23]. In LPS-stimulated BV2 cells, FA treatment dose-dependently reduced NO $(p<0.001)$ (Fig. 1E), IL-1 $\beta(p<0.001)$ (Fig. 1F), and IL-6 $(p<0.001)$ (Fig. 1G) production, indicative of anti-inflammatory properties.

\section{FA treatment ameliorated AD-like symptoms in APP/PS1 mice}

Six-week FA administration suppressed the high index levels in the liver $(p<0.001)$ (Fig. S2B) and kidneys $(p<0.01)$ (Fig. S2D) of APP/PS1 mice, without influencing their body weight (Fig. S2A) and spleen index (Fig. S2C). No evident pathological changes were observed in the liver (Fig. S3A), spleen (Fig. S3B), and kidney (Fig. S3C) after FA administration, supporting the safety of FA treatment in mice.

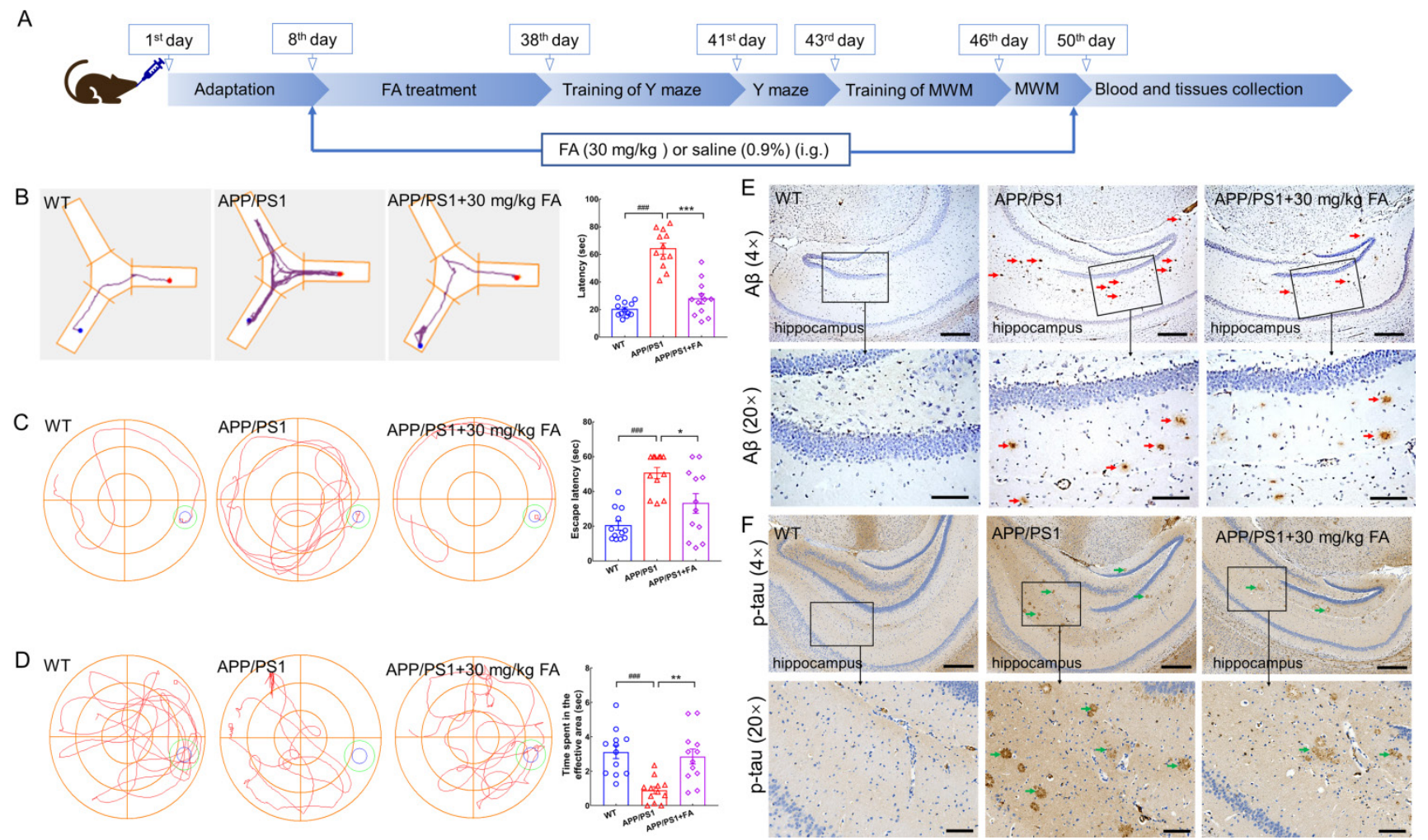

Figure 2. FA treatment ameliorates AD symptoms in APP/PS1 mice. (A) Schematic diagram of animal experiments. (B) FA treatment shortened foraging time of APP/PSI mice in the Y-maze test $(n=12)$. (C) FA treatment shortened the escape latency of APP/PS1 mice in the MWM navigation test $(n=12)$. (D) FA treatment increased the time of APP/PS1 mice spent in the effective area in the MWM probe trials (no platform) $(n=12)$. The blue and green circles indicate the position of the original platform and effective area, respectively. (E) FA treatment decreased $A \beta_{1-42}$ (red arrow) deposition in the hippocampus of APP/PSI mice ( $n=3$ ). $(F)$ FA treatment suppressed the levels of phosphorylated tau protein (green arrow) in the hippocampus of APP/PS1 mice $(n=3)$. Scale bars: $400 \mu \mathrm{m}$ for $4 \times$ magnification and $100 \mu \mathrm{m}$ for $20 \times$ magnification. The data are presented as mean \pm S.E.M. ${ }^{m} p<0.001$ vs. WT mice; ${ }^{*} p<0.05,{ }^{*} p<0.01$ and $* * * p<0.001$ vs. APP/PS1 mice. 


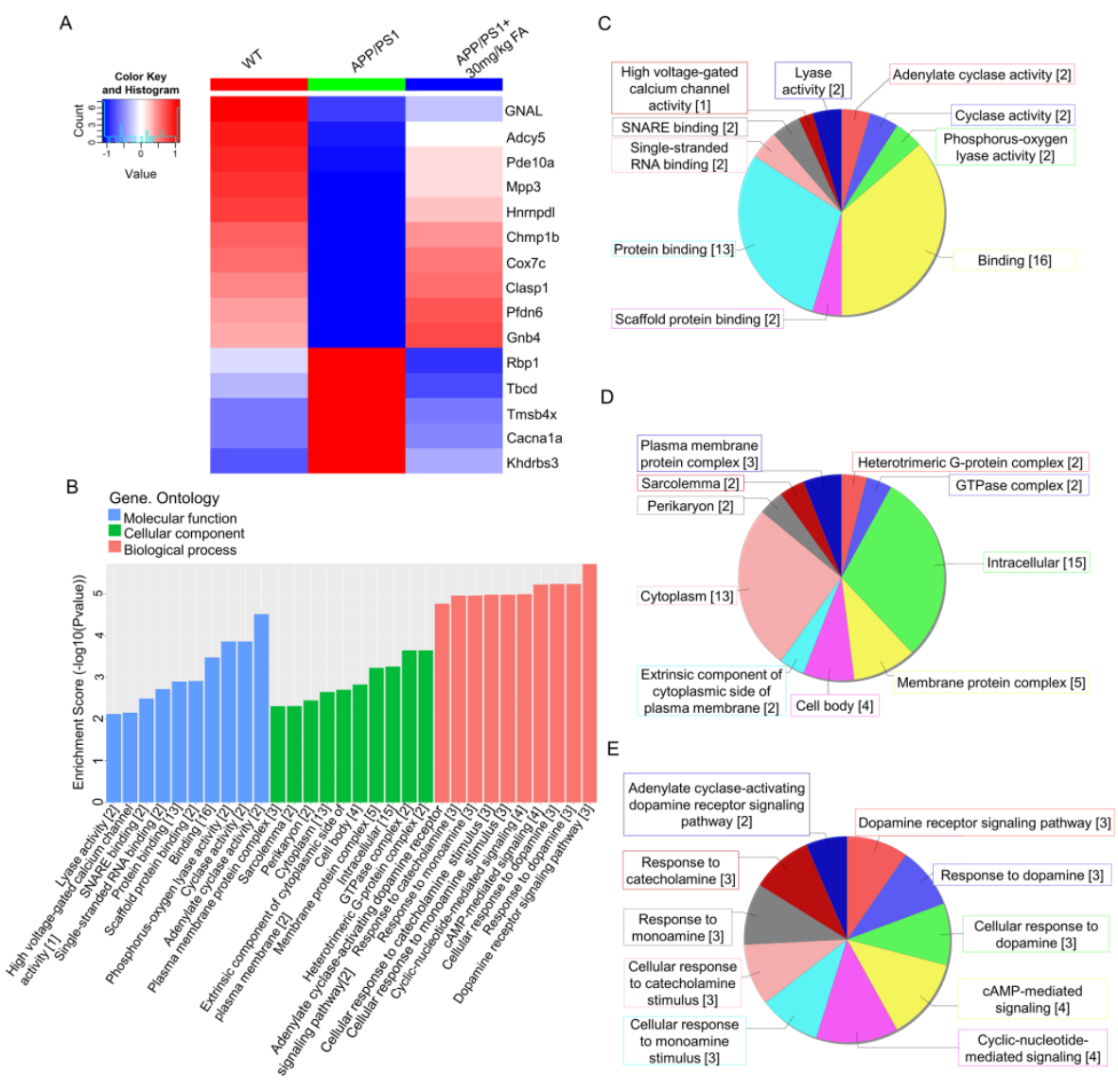

$\mathrm{F}$
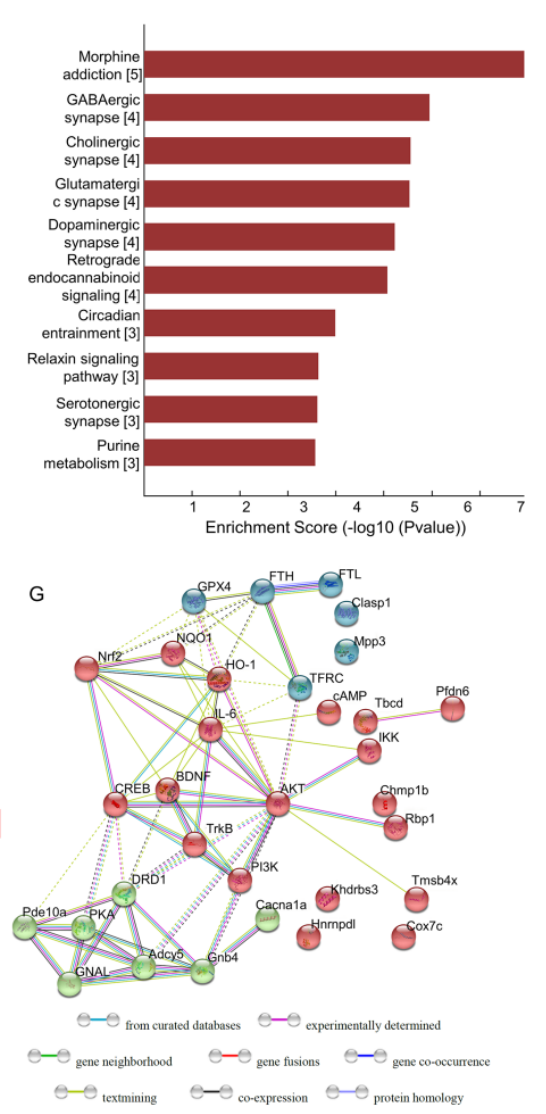

Figure 3. Proteomics and bioinformatics analysis of the hippocampus in APP/PS1 mice. (A) Heat map of 15 proteins. Red and blue indicate high-abundance and low-abundance proteins, respectively. (B) GO enrichment analysis of differentially expressed proteins among WT, APP/PS1, and FA-treated APP/PS1 mice. Molecular function, cellular component, and biological process are marked in blue, green, and pink, respectively. (C) Pie chart of GO molecular function classification. (D) Pie chart of GO cellular component classification. (E) Pie chart of GO biological process classification. (F) KEGG enrichment analysis of differentially expressed proteins among WT, APP/PS1, and FA-treated APP/PSI mice. Rows represent individual biological processes and the number of related altered proteins. (G) Protein-protein interaction network analysis generated using STRING software.

In the Y-maze test, the foraging time of APP/PS1 mice was longer than that of WT mice $(p<0.001)$. In contrast, foraging time was shortened after FA administration in APP/PS1 mice ( $p<0.001)$ (Fig. 2B). Memory and spatial recognition abilities of mice were assessed using the MWM. In APP/PS1 mice, FA treatment shortened the escape latency in the navigation test $(p<0.05)$ (Fig. 2 C) and increased the time spent in the effective area in the probe trials (no platform) $(p<0.01)$ (Fig. 2D).

$\mathrm{A} \beta$ deposition and neurofibrillary tangles formed by highly phosphorylated tau protein are considered the two major pathological hallmarks of AD [19]. Compared with vehicle treatment, FA treatment strongly suppressed $A \beta$ deposition (Fig. 2E) and high p-tau levels (Fig. 2F) in the hippocampus. These data suggested that FA treatment ameliorated AD-like symptoms in APP/PS1 mice.

\section{FA treatment augmented dopaminergic signaling and prevented ferroptosis in APP/PS1 mice}

Label-free proteomics was performed to screen proteins that were significantly differentially expressed among WT, APP/PS1, and FA-treated APP/PS1 mice. The analysis identified 15 proteins with significantly different expression (Fig. 3A and Table S2), among which, adenylate cyclase 5 (Adcy5) and guanine nucleotide-binding protein $G($ olf $)$ subunit a (GNAL) were associated with cyclic adenosine monophosphate (cAMP) production and dopaminergic downstream signaling pathways [24, 25]. GO analysis detected cyclase activity, cAMPmediated signaling, and dopamine (DA) receptor signaling pathways (Fig. 3B-E). KEGG analysis revealed that significantly differentially expressed proteins were associated with dopaminergic synapses (Fig. 3F). As a key neurotransmitter in the nervous system, DA prevents ferroptosis by reducing iron accumulation and degrading GPX4 in cells [26]. DA receptors inhibit NF-kB activity via the protein phosphatase 2A-dependent protein kinase B (AKT) pathway, thereby inhibiting inflammation [27]. Based on biosynthesis, the relationships among the 15 significant proteins, ferroptosis-related proteins, and neuroinflammation-related proteins are presented in 
the STRING interaction analysis (Fig. 3G), implying the regulation of ferroptosis and neuroinflammation by FA.

To further verify the proteomics results, immunohistochemistry was performed to detect the expression levels of cAMP, p-AKT, and GPX4 in the mouse brain. The expression of cAMP $(p<0.01)$ (Fig. $4 \mathrm{~A}$ and Fig. S4A), p-AKT $(p<0.05)$ (Fig. $4 \mathrm{~B}$ and Fig. S4B), and GPX4 ( $p<0.01)$ (Fig. 4C and Fig. S4C) in the cerebral cortex was significantly increased in FA-treated APP/PS1 mice compared to that in vehicle-treated APP/PS1 mice.

Proteins related to the dopaminergic system and ferroptosis were analyzed. Compared with vehicletreated APP/PS1 mice, FA-treated APP/PS1 mice exhibited higher levels of dopamine receptor D1 (DRD1) (a family of dopamine receptors that lead to protein kinase A (PKA) activation [28]; $p<0.05$ ), GNAL (encoding $\mathrm{Ga}(\mathrm{olf})$, which is related to increased cAMP levels [29]; $p<0.01$ ), Adcy5 (the main downstream effector of DA receptor signaling that is highly expressed in the striatum [24]; $p<0.05)$, cAMP (a highly conserved second messenger that participates in DA-mediated learning [24]; $p<0.001$ ), and phospho (p)-PKA (a kinase positively coupled with dopamine D1 receptors that protects hippocampal neurons [28]); $p<0.05$ ) (Fig. 4D and Fig. S4D). These proteins are all related to the regulation of the dopaminergic system. Further, FA treatment increased the expression levels of p-cAMP-response element binding protein (CREB) (a protein that is essential for the formation of hippocampal-dependent long-term memory [30]; $p<0.001$ ), brain-derived neurotrophic factor (BDNF) (a downstream target gene of CREB that supports neuronal survival and promotes synaptic transmission [30]; $p<0.01$ ), p-tyrosine kinase receptor B (TrkB) (a downstream target gene of BDNF that protects neurons and synapses in its phosphorylated form [30]; $p<0.001)$, p-phosphatidylinositol-3-kinase (PI3K) (an upstream regulator of AKT kinase [31]; $p<0.05$ ), and p-AKT (a proven target of ferroptosis regulation [31]; $p<0.05$ ) in the brains of APP/PS1 mice (Fig. 4E and Fig. S4E), implicating the regulation of key proteins in the dopaminergic terminal signaling pathway.
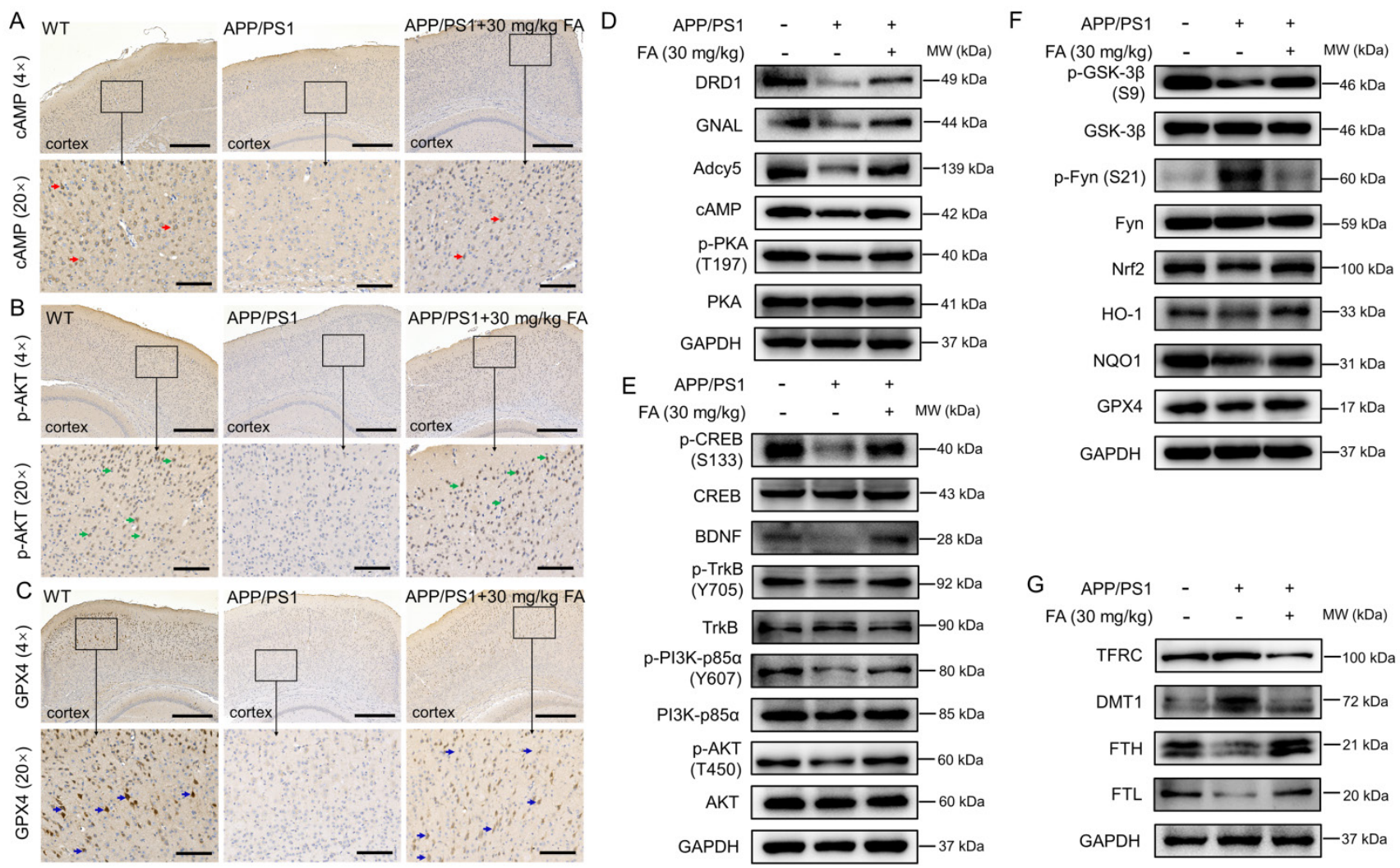

Figure 4. FA treatment regulates the dopaminergic system and ferroptosis in APP/PS1 mice. FA treatment increased the expression of (A) cAMP (red arrows), (B) P-AKT (green arrows), and (C) GPX4 (blue arrows) in the cortex in APP/PS1 mice $(n=3$ ). Scale bar: $400 \mu \mathrm{m}$ for $4 \times$ magnification and $100 \mu \mathrm{m}$ for $20 \times$ magnification. (D) FA treatment enhanced the expression levels of DRD1, GNAL, Adcy5, cAMP, and p-PKA in the brains of APP/PS1 mice $(n=3)$. (E) FA treatment upregulated the phosphorylation of CREB, TrkB, PI3K, and AKT, and BDNF expression in the brains of APP/PS1 mice $(n=3)$. (F) FA treatment suppressed the expression of $p$-Fyn and upregulated the expression of $\mathrm{p}$-GSK-3 $\beta, \mathrm{GPX} 4, \mathrm{Nrf2}$, and downstream proteins in the brains of APP/PS1 mice $(n=3)$. (G) FA treatment suppressed the expression of TFRC and DMT1 and upregulated the expression of FTH, and FTL in the brains of APP/PS1 mice $(n=3)$. 
Nrf2 signaling can protect dopaminergic neurons against ferroptosis [32]. Compared with vehicle-treated APP/PS1 mice, FA-treated APP/PS1 mice exhibited reduced phosphorylation of Fyn (its phosphorylated form accumulates in the nucleus and phosphorylates Nrf2 at tyrosine 568 [33]; $p<0.001$ ) and enhanced expression levels of GPX4 $(p<0.001)$, p-glycogen synthase kinase-3 $\beta$ (GSK-3 $\beta$ ) (an upstream regulator of Fyn kinase in the control of nuclear export of Nrf2 [33]; $p<0.01), \operatorname{Nrf2}(p<0.05)$, and its downstream proteins $(p<0.01)$ (Fig. $4 \mathrm{~F}$ and Fig. S4F), confirming the modulation of Nrf2 signaling.

FA treatment strongly suppressed the expression of transferrin receptor (TFRC) (a membrane receptor that mediates $\mathrm{Fe}^{3+}$ transport into cells [8]; $p<0.001$ ) and divalent metal-ion transporter-1 (DMT1) (a protein that transports $\mathrm{Fe}^{2+}$ into the cytoplasm [8]; $p<$ 0.05 ) and increased the expression of ferritin heavy chain $(\mathrm{FTH})$ (a peptide responsible for capturing iron ions [34]; $p<0.001$ ), and ferritin light chain (FTL) (a peptide responsible for iron storage in cells [35]; $p<$ 0.001) in the brains of APP/PS1 mice (Fig. 4G and Fig. $\mathrm{S} 4 \mathrm{G})$, which further confirmed the alleviation of ferroptosis by FA treatment.

\section{FA treatment mitigated neuroinflammation in APP/PS1 mice}

In $\mathrm{AD}$, neuroinflammation aggravates $A \beta$ and tau pathology [11]. The release of lipid metabolites in ferroptosis triggers inflammation, which reciprocally promotes ferroptosis by increasing iron deposition [36]. FA treatment strongly suppressed the expression of ionized calcium-binding adapter molecule 1 (Iba1) (a microglial marker [6]) and glial fibrillary acidic protein (GFAP) (an astrocytic marker [6]) in the hippocampus $(p<0.001)$ (Fig. 5A, C and Fig. S5A, C) and cerebral cortex $(p<0.01)$ (Fig. 5B, D and Fig. S5B, D) in APP/PS1 mice. Double-labeling with GFAP and S100 calcium-binding protein B (S100B) further confirmed the inhibitory effect of FA treatment on the activation of astrocytes (Fig. S6).

An increase in pro-inflammatory cytokine levels and a decrease in anti-inflammatory cytokine levels exacerbate the degree of dementia in $\mathrm{AD}$ [37]. Additionally, high expression of MCP-1 is observed in microglia surrounding senile plaques in the brains of patients with $\mathrm{AD}$ [38]. ELISA results revealed a marked increase in TGF- $\beta$ expression $(p<0.01)$ (Fig. $5 \mathrm{E})$ and decreased MCP-1 $(p<0.01)$ (Fig. 5F), IL-1 $(p$ $<0.01$ ) (Fig. 5G), IL-6 ( $p<0.01)$ (Fig. 5H), and TNF-a $(p$ $<0.01$ ) (Fig. 5I) expression levels in the brains of FA-treated APP/PS1 mice relative to those of vehicle-treated APP/PS1 mice.

Compared to WT mice, APP/PS1 mice exhibited higher levels of Iba1 $(p<0.05)$, GFAP $(p<0.01)$, arachidonate 5-lipoxygenase (ALOX5) (an enzyme that participates in AD-related neuroinflammation by enhancing Iba1 and GFAP [39]; $p<0.001)$, myeloid cell surface antigen CD33 (CD33) (a transmembrane sialic acid-binding receptor located on the surface of microglia that promotes $A \beta$ pathology $[40] ; p<0.05)$, and inducible nitric oxide synthase (iNOS) (an enzyme that produces $\mathrm{NO}$ which can be toxic to neurons and is upregulated in the brains of patients with AD [37]; $p<0.001$ ) (Fig. 5J and Fig. S5E). FA treatment markedly decreased the expression levels of these proteins $(p<0.05)$ (Fig. 5J and Fig. S5E).

Furthermore, FA treatment induced an upregulation of anti-inflammatory factors, including IL-4 $(p<0.01)$ and IL-10 $(p<0.001)$ and a concomitant downregulation of proinflammatory factors, including IL-1 $\beta(p<0.01)$, IL-6 $(p<0.01)$, IL-18 $(p<$ $0.01)$, and TNF-a $(p<0.01)$, as well as p-IкB kinase (IKK) $(p<0.05)$, p-IкB $(p<0.01)$, and p-NF-кB $(p<$ 0.01) (Fig. 5K, L and Fig. S5F, G). Collectively, these data suggested that FA treatment suppressed neuroinflammation in the brains of APP/PS1 mice.

\section{FA treatment attenuated ferroptosis and neuroinflammation in erastin-exposed HT22 cells}

As a classic ferroptosis inducer, erastin changes the permeability of the outer mitochondrial membrane by binding voltage-dependent anion channel $2 / 3$ to reduce the rate of nicotinamide adenine dinucleotide oxidation, thereby inducing ferroptosis [41]. Further, the inhibition of GPX4 increases lipid ROS production, eventually leading to cell ferroptosis [42]. FA pretreatment significantly improved cell viability in erastin-exposed HT22 cells $(p<0.001)$ (Fig. 6A). NAD(P)H-GSH-GPX4 signaling is a classic pathway that inhibits ferroptosis. GSH acts as an electron donor to reduce toxic phospholipid hydroperoxide to non-toxic phospholipid alcohol. This maintains GPX4 activity and reduces lipid peroxidation which can be broken down into MDA, an end product that is severely toxic to cells [8]. FA treatment significantly suppressed MDA levels $(p<$ 0.001) (Fig. 6B) and increased GSH levels $(p<0.001)$ (Fig. 6C) in erastin-stimulated HT22 cells. BODIPY 581/591 C11 staining revealed that FA treatment inhibited erastin-induced lipid ROS production in HT22 cells $(p<0.01)$ (Fig. 6D and Fig. S7).

Ferroptosis results in morphological changes in mitochondrial ultrastructure [43]. TEM analysis revealed that mitochondria in erastin-stimulated cells had smaller volume, higher electron density, and disrupted mitochondrial cristae; these pathological alterations in morphology were ameliorated by FA treatment (Fig. 6E). In erastin-exposed HT22 cells, FA 
treatment significantly attenuated $\mathrm{p}$-Fyn expression $(p$ $<0.05)$ and upregulated p-PI3K $(p<0.05)$, p-AKT $(p<$ $0.01)$, p-GSK-3 $\beta(p<0.05)$, Nrf2 $(p<0.05)$, and $\mathrm{NAD}(\mathrm{P}) \mathrm{H}$ quinone dehydrogenase 1 (NQO1) $(p<$ 0.05 ) expression (Fig. 6F and Fig. S8A). Similar to the in vivo data, FA treatment upregulated the expression of GPX4 $(p<0.05)$, cystine/glutamate transporter $(\mathrm{xCT})(p<0.01)$, FTH $(p<0.05)$, FTL $(p<0.05)$, and ferroportin (FPN) (the only known protein in mammals that underscores export of iron [8]; $p<0.05$ ) and suppressed the expression of TFRC $(p<0.05)$ and DMT1 $(p<0.05)$ (Fig. 6G and Fig. S8B). FA treatment suppressed the activation of NF-KB signaling $(p<$ $0.05)$, which further regulated the expression of ILs ( $p$ $<0.05)$ and TNF-a $(p<0.05)$ (Fig. 6H and Fig. S8C). In conjunction with the in vivo results, these findings indicated that FA treatment exerted anti-ferroptosis and anti-neuroinflammatory effects.

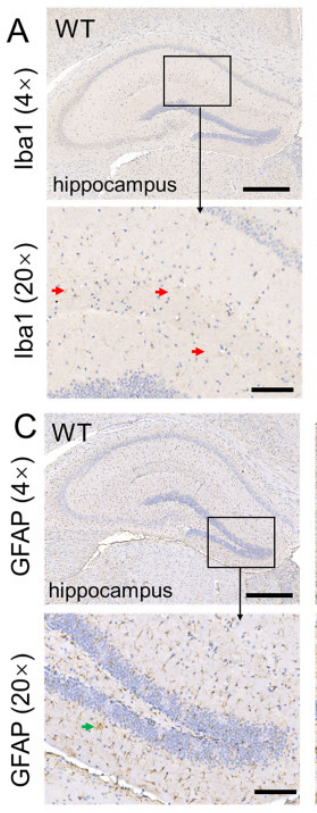

E

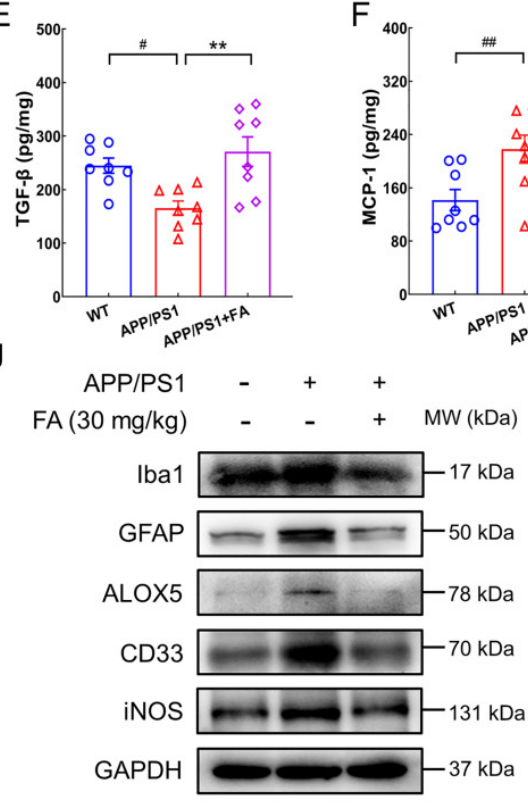

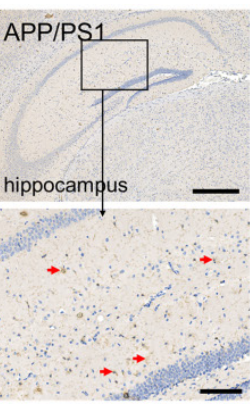
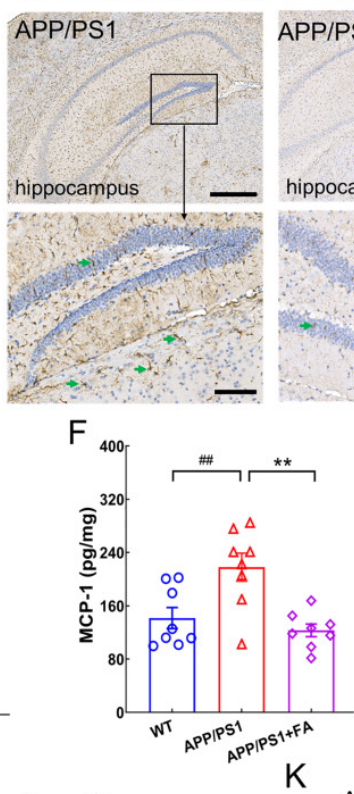

$\mathrm{K}$
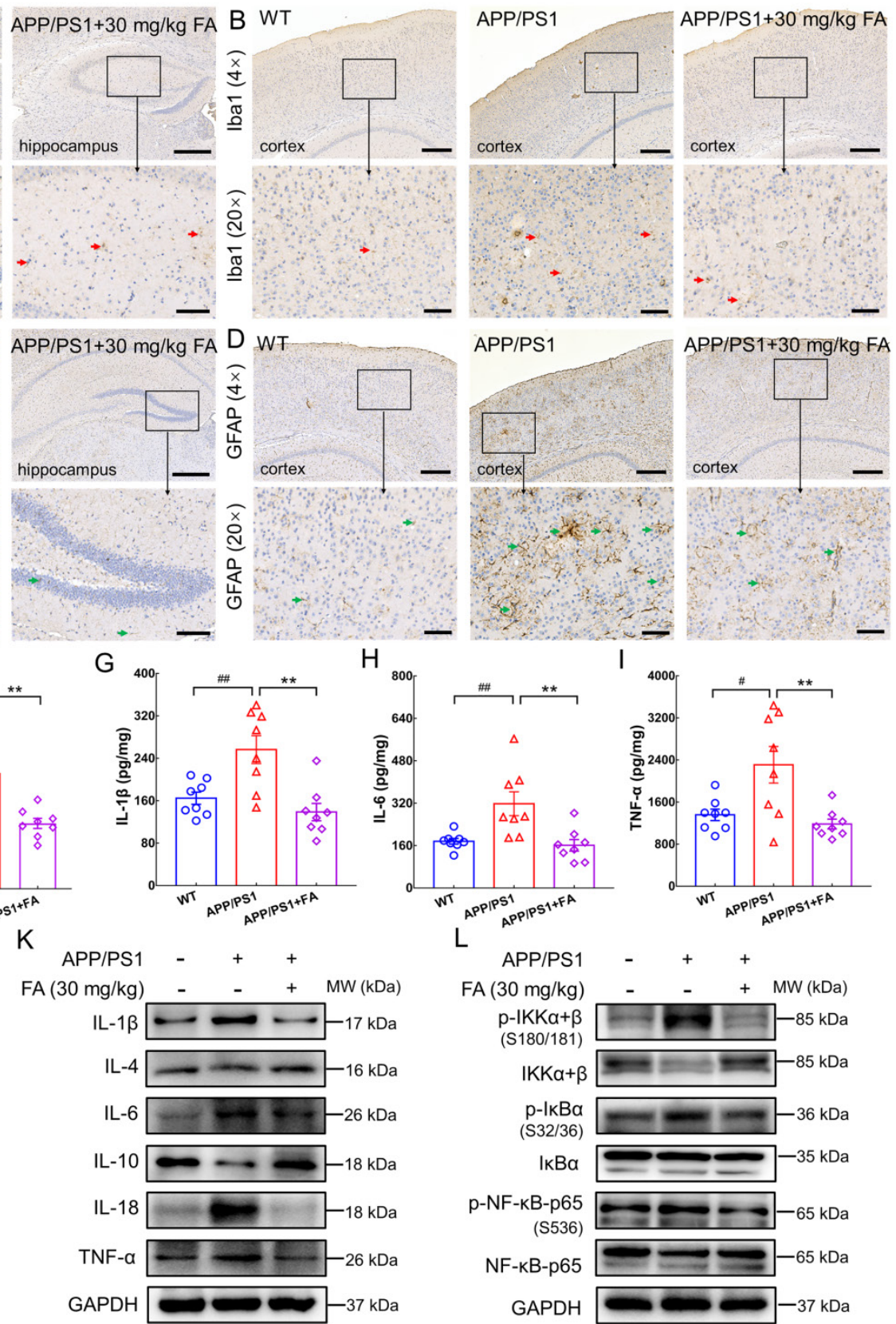

Figure 5. FA treatment alleviates neuroinflammation in APP/PSI mice. FA treatment decreased the expression levels of Ibal (red arrows) in the (A) hippocampus and $(\mathbf{B})$ cerebral cortex in APP/PS1 mice $(n=3)$. FA treatment decreased the expression levels of GFAP (green arrows) in the (C) hippocampus and (D) cerebral cortex in APP/PS1 mice $(n=3)$. Scale bar: $400 \mu \mathrm{m}$ for $4 \times$ magnification and $100 \mu \mathrm{m}$ for $20 \times$ magnification. FA treatment upregulated the expression of (E) TGF- $\beta$ and suppressed the expression levels of $(\mathbf{F})$ MCP-1, (G) IL-1 $\beta,(\mathbf{H})$ IL-6, and (I) TNF- $\alpha$ in the brains of APP/PS1 mice $(n=8)$ detected with ELISA. (J) FA treatment downregulated the expression of Iba 1, GFAP, ALOX5, CD33, and iNOS in the brains of APP/PS1 mice $(n=3)$. (K) FA treatment ameliorated the levels of ILs and TNF- $\alpha$ in the brains of APP/PS1 mice $(n=3)$. (L) FA treatment suppressed the phosphorylation of IKK, IKB, and NF-KB in the brains of APP/PS1 mice $(n=3)$. The data are presented as mean \pm S.E.M. \# $p<0.05$, \#\# $<0.01$ vs. WT mice; $* * p<0.01$ vs. APP/PS1 mice. 
A

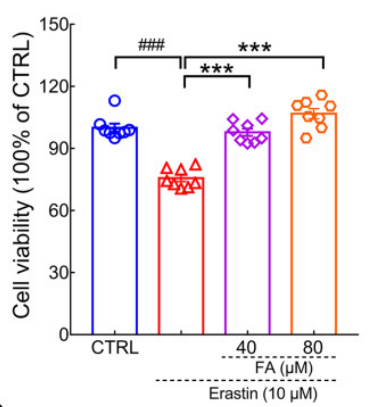

B

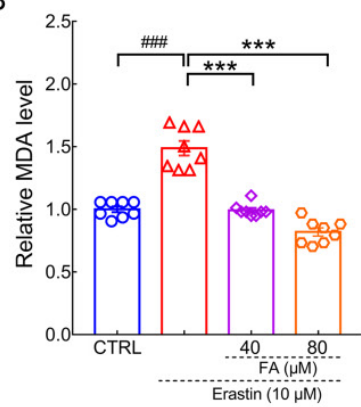

C

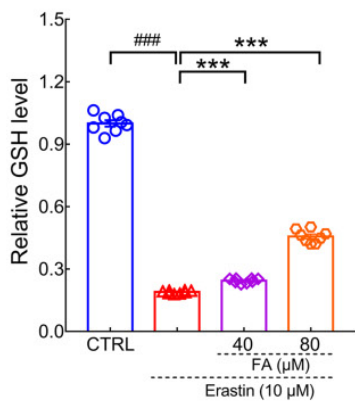

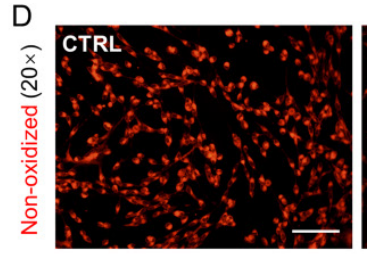
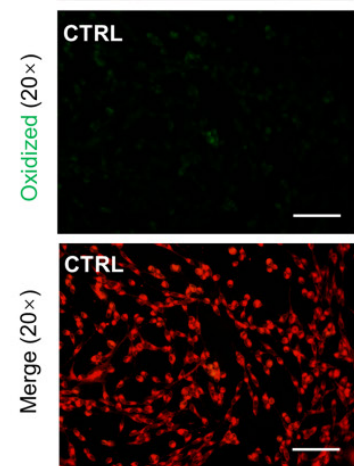

E

峞

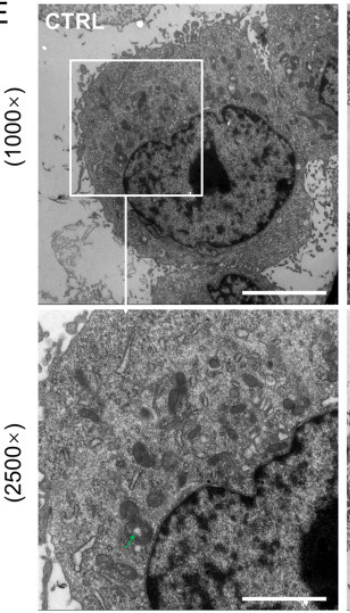

G
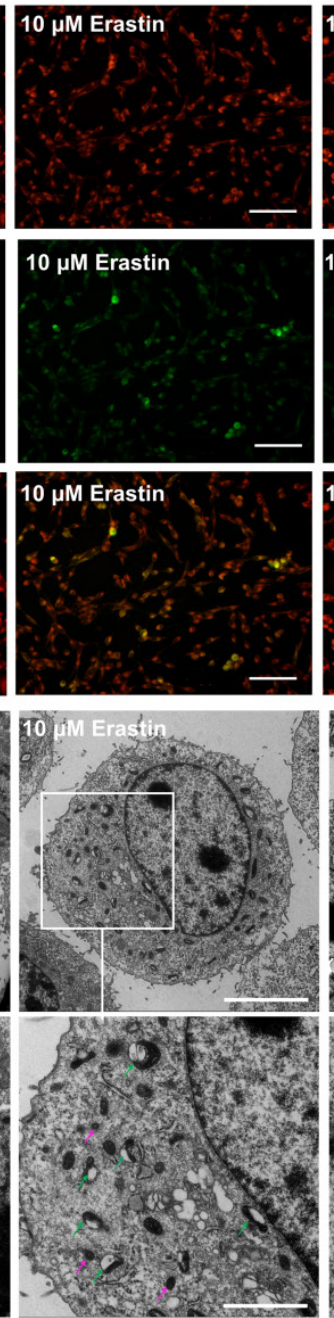

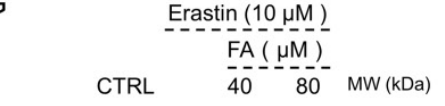

GPX4 $-17 \mathrm{kDa}$

$\mathrm{xCT} \longrightarrow-35 \mathrm{kDa}$

TFRC $=-100 \mathrm{kDa}$

DMT1 $---72 \mathrm{kDa}$

$\mathrm{FTH}=-21 \mathrm{kDa}$

$\mathrm{FTL}---20 \mathrm{kDa}$

$\mathrm{FPN}=-\mathbf{- 2}$-63 kDa

GAPDH $-37 \mathrm{kDa}$
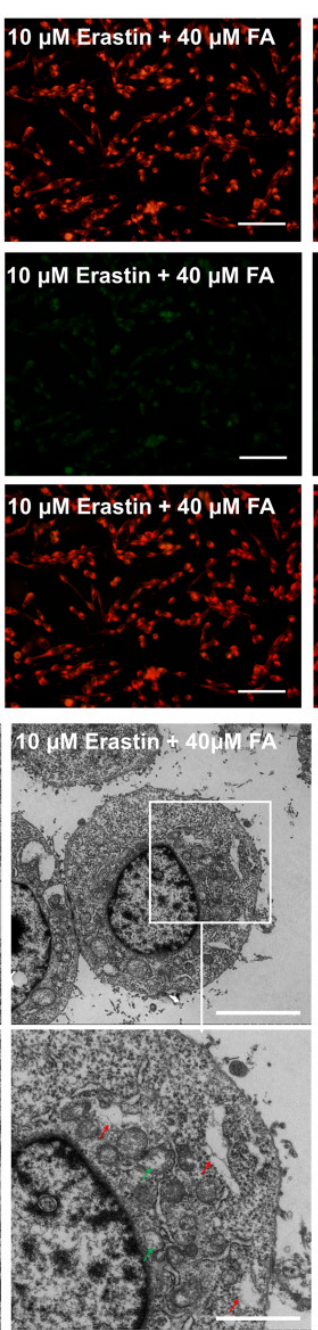

$\mathrm{H}$

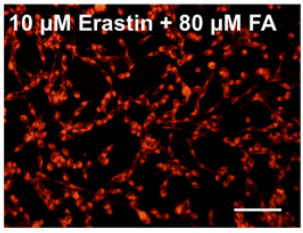

$10 \mu \mathrm{M}$ Erastin $+80 \mu \mathrm{M}$ FA

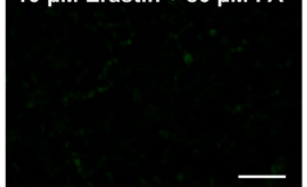

10 $\mu$ M Erastin $+80 \mu \mathrm{M} \mathrm{FA}$
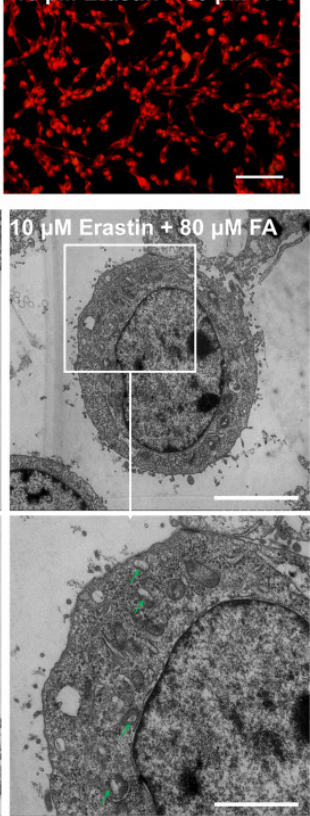

Erastin $(10 \mu \mathrm{M})$ $\mathrm{FA}(\mu \mathrm{M})$

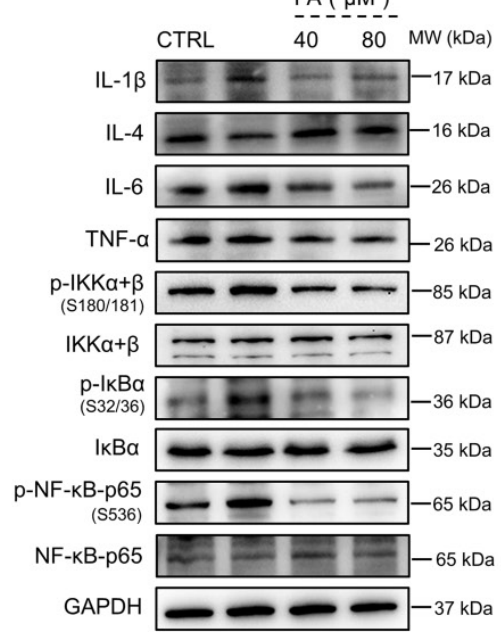

Figure 6. FA alleviates ferroptosis-related inflammation in erastin-exposed HT22 cells. FA treatment (A) improved cell viability, (B) decreased MDA levels, and (C) increased GSH levels in erastin-exposed HT22 cells $(n=8)$. (D) FA treatment suppressed lipid peroxidation in erastin-exposed HT22 cells $(n=3)$ as determined using BODIPY $581 / 591 \mathrm{C11}$. Red and green fluorescence signals represent non-oxidized and oxidized states, respectively. Scale bar: $100 \mu \mathrm{m}$. (E) Ultrastructure of HT22 cells in each group was analyzed using TEM $(n=3)$. Green, pink, and red arrows indicate disrupted mitochondrial cristae, increased mitochondrial electron density, and rough endoplasmic reticulum expansion. Scale bar: $5 \mu \mathrm{m}$ for $1000 \times$ magnification and $2 \mu \mathrm{m}$ for $2500 \times$ magnification. (F) FA treatment upregulated the expression of p-PI3K, p-AKT, p-GSK-3 3 , Nrf2, and NQO1, and downregulated the expression of p-Fyn in erastin-exposed HT22 cells $(n=3)$. (G) FA treatment upregulated the expression of GPX4, xCT, FTH, FTL, and FPN, and suppressed the expression of TFRC and DMT 1 in erastin-exposed HT22 cells $(n=3)$. (H) FA treatment downregulated the expression of IL-1 $\beta$, IL- 6 , and TNF- $\alpha$; suppressed the phosphorylation of IKK, IKB, and NF-KB; and upregulated the expression of IL-4 in erastin-exposed HT22 cells $(n=3)$. The data are presented as mean \pm S.E.M. CTRL HT22 cells; **** $<0.001$ vs. erastin-exposed HT22 cells. 
A

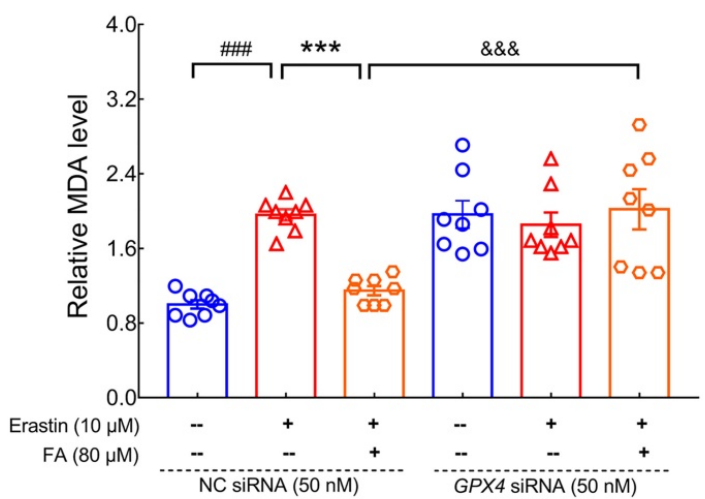

B

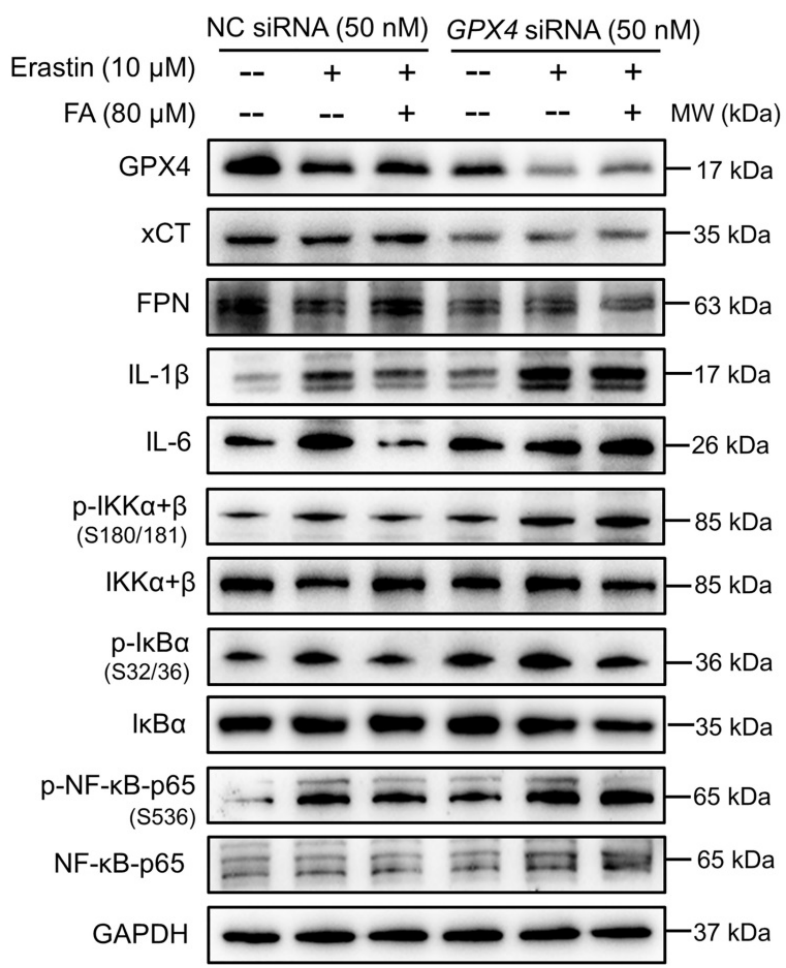

C

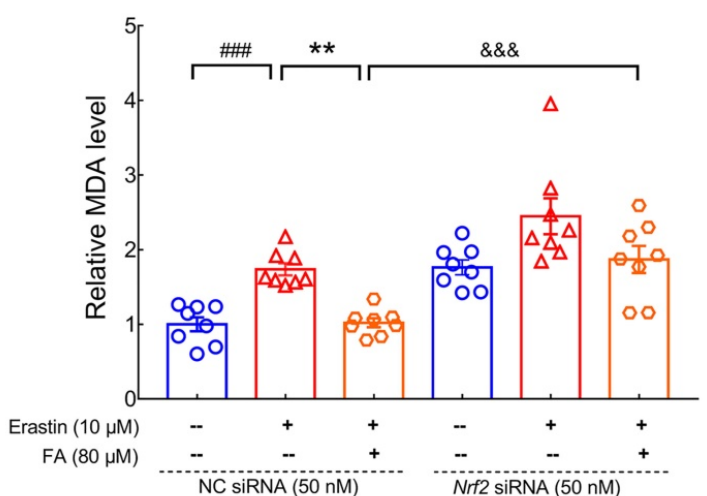

D

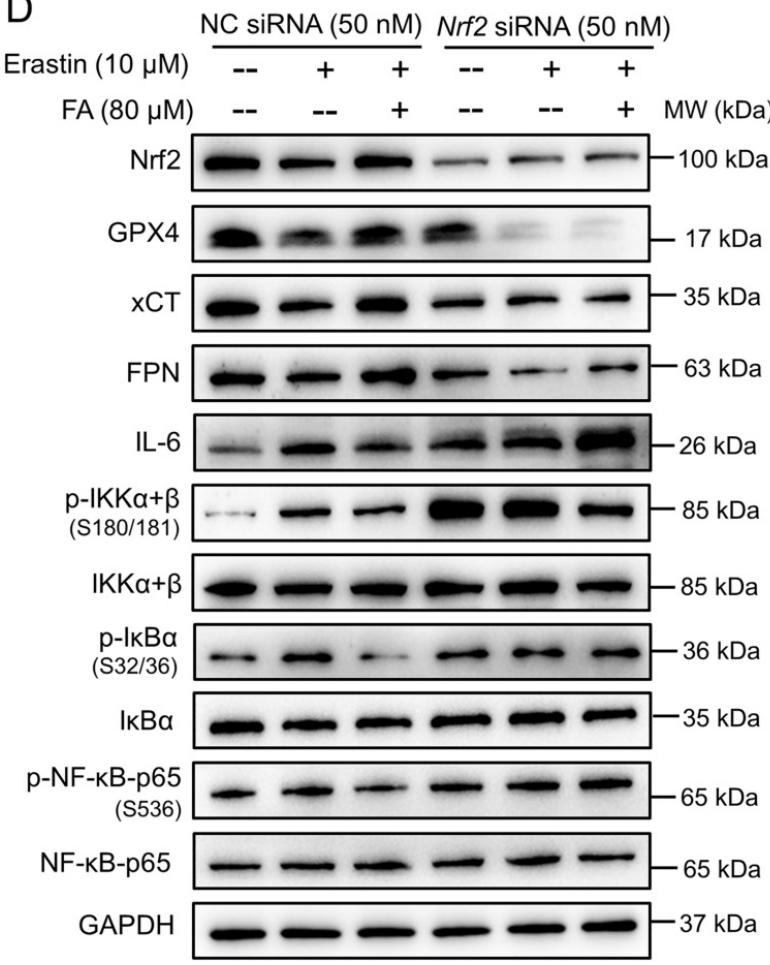

Figure 7. GPX4 and Nrf2 are involved in the anti-ferroptosis and anti-neuroinflammatory effects of FA treatment. (A) FA treatment-induced decreases in MDA levels were blocked in GPX4 siRNA-transfected erastin-exposed HT22 cells $(n=8)$. (B) FA treatment-induced increases in GPX4, $x$ CT and FPN levels; reduction in IL-6 and IL-1 $\beta$ levels; and suppression of NF-KB signaling were abolished in GPX4 siRNA-transfected erastin-exposed HT22 cells $(n=3)$. (C) FA treatment-induced decreases in MDA levels were blocked in Nrf2 siRNA-transfected erastin-exposed HT22 cells $(n=8)$. (D) FA treatment-induced upregulation of Nrf2, GPX4, xCT, and FPN was abolished and NF-KB signaling was activated in Nrf2 siRNA-transfected erastin-exposed HT22 cells $(n=3)$. The data are presented as mean \pm S.E.M. NC siRNA; **p $<0.01$, ***p $<0.001$ vs. erastin-exposed HT22 cells transfected with NC siRNA; \&\&\& $<0.001$ vs. FA-treated HT22 cells transfected with NC siRNA.

\section{Anti-ferroptosis and anti-neuroinflammatory effects of FA treatment were mediated by the Nrf2/GPX4 axis}

Increased iron levels in the brain induce pro-inflammatory factor production by microglia; conversely, iron accumulation is an established consequence of inflammation [7]. Nevertheless, the specific regulatory processes linking neuroinflammation and ferroptosis have yet to be elucidated. A recent study reported that targeting the Nrf2/GPX4 axis effectively regulated ferroptosis [44]. GPX4 plays a key role in preventing ferroptosis by reducing phospholipid hydroperoxide, thus inhibiting lipid peroxidation. Accordingly, GPX4 activation inhibits
NF-kB signaling in lipid peroxidation-mediated diseases [13]. The regulatory effects of FA treatment on MDA levels $(p<0.001)$ (Fig. 7A); expression of GPX4 $(p<0.001), x C T(p<0.05)$, FPN $(p<0.01)$, and ILs $(p<0.01)$; and IKK/ІкB/NF-кB activation $(p<$ $0.05)$ were all strongly abolished in GPX4 siRNA-transfected erastin-exposed HT22 cells (Fig. 7B and Fig. S9). Furthermore, the molecular docking results showed that FA possessed an affinity for GPX4 with a binding energy of $-6.35 \mathrm{kcal} / \mathrm{mol}$, FA formed hydrogen bonds with GPX4 via GLN-45, CYS-46, GLY-47, LYS-48, ARG-80, and GLN-81. The interaction between FA and GPX4 formed a binding pocket (Fig. S10). 
$\mathrm{Nrf2}$ is a negative regulator of $A \beta$ pathology [45] and modulates ferroptosis by regulating endogenous anti-ferroptosis components, including upregulation of $\mathrm{xCT}$, activation of $\mathrm{FTH}$, and heme oxygenase- 1 (HO-1), and restoration of GPX4 activity [12]. The regulatory effects of FA treatment on MDA levels $(p<$ $0.001)$ (Fig. 7C); expression of Nrf2 $(p<0.001)$, GPX4 $(p<0.001), x \mathrm{xT}(p<0.01), \mathrm{FPN}(p<0.001)$, and IL-6 ( $p$ $<0.01)$; and IKK/IкB/NF-kB activation $(p<0.01)$ were all strongly abolished in Nrf2 siRNA-transfected erastin-exposed HT22 cells (Fig. 7D and Fig. S11). These data are consistent with previous observations that Nrf2 regulates ferroptosis and neuroinflammation by influencing GPX4 expression. Collectively, these data confirmed the involvement of the Nrf2/GPX4 axis in FA-mediated anti-ferroptosis and anti-neuroinflammation and provided insight into the crosstalk between ferroptosis and neuroinflammation.

\section{Discussion}

In this study, we first expounded the protective properties of $\mathrm{FA}$ in $\mathrm{AD}$ based on its effects in A $\beta$-exposed N2a cells, erastin-stimulated HT22 cells, and LPS-stimulated BV2 cells as well as its beneficial effects on memory and cognitive abilities in APP/PS1 mice with AD-like behaviors. Label-free quantitative proteomics analysis demonstrated that FA treatment modulates the expression of Adcy5 and GNAL, which affect learning by altering cAMP-related dopamine signaling [24, 25], subsequently suppressing neuroinflammation and preventing ferroptosis by inhibiting NF-KB activity and reducing iron accumulation [26, 27]. Furthermore, the Nrf2/GPX4 axis was confirmed to play key roles in FA-mediated anti-neuroinflammation and anti-ferroptosis.

To date, the pathogenesis of $\mathrm{AD}$ has not been fully elucidated, and the development of effective treatments has been challenging [3]. Natural products, such as marine-derived oligosaccharides (GV-971), have been identified as potential therapeutic agents [46]. Extracellular senile plaques composed of $A \beta$ peptides have been reported as a key marker of the initiation of the pathogenic cascade of $\mathrm{AD}$ [47], and an imbalance between production and clearance may induce synaptic dysfunction and neuronal damage [48]. The monoclonal antibody aducanumab targets soluble and insoluble neurotoxic $A \beta$ oligomers and was approved by the US FDA for the treatment of AD in June 2021 [5]. Activated microglia associated with $\mathrm{A} \beta$ neurotoxicity cause chronic inflammation and aggravate neuronal damage [49]. GV-971 has been reported to inhibit intestinal dysbiosis and regulate the accumulation of phenylalanine/isoleucine and suppress neuroinflammation, thereby improving cognitive impairment, which confirms the key role of neuroinflammation in the pathological process underscoring $\mathrm{AD}$ [46]. FA treatment reduced $\mathrm{A} \beta$ deposition and exhibited anti-neuroinflammatory effects, as demonstrated by the suppression of pro-inflammatory cytokines and enhancement of anti-inflammatory cytokines in several cell lines and APP/PS1 mice.

Significant reductions in DA and DA metabolite levels have been observed in several regions of the post-mortem AD brain [50]. These changes are associated with behavioral symptoms such as apathy and depression and may contribute to cognitive decline [51]. In the Tg2576 mouse model of AD, age-dependent alterations in dopaminergic neuron loss in the ventral tegmental area occur prior to the formation of amyloid plaques, leading to memory deficits [52]. Rotigotine, a DA agonist, has demonstrated beneficial effects on frontal-executive function in a phase 2 trial for $\mathrm{AD}$ [4]. The selective DRD1 agonist SKF81297 is thought to be involved in ameliorating hippocampal synaptic damage and improving spatial memory in AD mouse models [53]. DRD1 is the most abundant DA receptor in the central nervous system, and its downstream signaling involves the binding of Gaolf, encoded by the GNAL gene, to guanosine triphosphate. This results in Adcy5 activation and subsequent cAMP production [25]. Allosteric activation of cAMP promotes cAMPdependent PKA phosphorylation of CREB [54], thereby initiating the transcription and translation of CREB target genes, which are essential for the formation of long-term memories [55]. Impaired cAMP signaling (including p-CREB) has been observed in the brains of $\mathrm{AD}$ patients and relevant mouse models [30, 56]. The enhancement of phosphorylated CREB ameliorates memory impairments in AD mouse models [57], which is consistent with the results of our study. Moreover, CREB mediates the formation of short-term memories by upregulating BDNF [58]. Accordingly, the combination of BDNF and its endogenous receptor, TrkB, may cause autophosphorylation of the intracellular domain of tyrosine residues, which initiates PI3K/AKT activation and promotes neuronal survival [59]. The present data confirm the involvement of dopaminergic signaling in FA-mediated anti-AD effects.

BDNF has been proposed to activate Nrf2 in astrocytes via the truncated TrkB.T1 and p75NTR receptor complex in astrocytes to protect dopaminergic neurons from ferroptosis [32]. The non-oxidized form of DA serves as a strong inhibitor of ferroptosis by inhibiting intracellular iron accumulation and iron-mediated ROS production or by reducing GSH consumption and GPX4 
degradation [26]. Intracellular iron is stored in ferritin, which is composed of FTH and FTL, or exported by FPN. Imbalances in iron input, storage, and output may affect susceptibility to lipid peroxidation $[8,60]$. During this process, TFRC and DMT1 regulate the translocation of iron into cells [61]. Due to the enrichment of polyunsaturated fatty acid in the brains of AD patients [60], lipid ROS alter the structure of the lipid bilayer and destroys its barrier function. The formation of lipid peroxides further amplifies ROS signals and decomposes these products to produce the toxic derivative, MDA. MDA reacts with DNA bases or proteins, causing severe cytotoxicity. GPX4 inhibits lipoxygenase-mediated lipid peroxidation by reducing phospholipid hydrogen peroxide and plays a key role in preventing ferroptosis [8]. In this study, FA treatment significantly reduced intracellular iron content by partially reducing iron entry and promoting iron output. These effects were associated with a reduction in lipid peroxidation in cell lines and APP/PS1 mice.

In pathological conditions of neurodegenerative diseases, iron overload triggers microglial polarization to the pro-inflammatory (M1) phenotype via ROS, which increases the secretion of TNF- $\alpha$ and IL-1 $\beta$ and promotes neuroinflammation [62]. IL-1 $\beta$ and TNF- $\alpha$ upregulate TFRC and DMT1 and downregulate FPN, resulting in increased ferrous iron influx and reduced iron efflux in cells [63]. Consequently, neuroinflammation and iron interact to form an amplified ROS production circuit, leading to neuronal ferroptosis [62]. Metabolic dysfunction of microglia and astrocytes leads to $A \beta$ accumulation, which in turn activates microglia and astrocytes, and activated glial cells release neuroinflammatory mediators, including IL-1 $\beta$ and IL-6 via Toll-like receptors and NF-kB signaling pathways [64, 65]. IL-1 $\beta$ induces IL-6 production by astrocytes and neurons, which in turn modulates behavior and cognitive function in rats administered with IL-6 into the bilateral hippocampus and in IL-6 knockout mice [66]. Our results demonstrated that FA treatment exerted anti-neuroinflammatory properties by targeting microglia and astrocytes via IKK/ІкB/ NF- $\mathrm{kB}$ signaling.

Based on previous research demonstrating the involvement of anti-ferroptosis and anti-neuroinflammation in $\mathrm{AD}$ treatment, we investigated FA-mediated anti-AD effects. GPX4 stimulates the release of anti-inflammatory lipid mediators in the inflammation-related arachidonic acid metabolic network and inhibits the NF-kB pathway activated by TNF or IL-1 [67]. An increase in GFAP expression levels was observed in adult mice that were deficient in GPX4 [10], which is consistent with our observation that GPX4 is a promising target for suppressing inflammation. Nrf2 has been reported to regulate neuroinflammation and ferroptosis and influence GPX4 expression [9]. siRNA-induced silencing of Nrf2 expression blocked melatonin-induced upregulation of GPX4 and xCT expression in MC3T3 cells [68]. Moreover, the concentration of supernatant TNF- $\alpha$ was increased and that of supernatant IL-4 and IL-10 was decreased in Nrf2-silenced BV2 cells [69]. These findings are consistent with the observation that Nrf2 siRNA abrogated FA-mediated upregulation of GPX4, $\mathrm{xCT}$, and FPN and NF-kB pathway inactivation. Collectively, these results indicate that FA treatment protects against erastin-induced ferroptosis by activating the Nrf2/GPX4 axis, and its suppression of ferroptosis exerts positive regulatory effects on neuroinflammation.

This study has several limitations. We only measured ferroptosis and neuroinflammation-related proteins and factors in the brains of APP/PS1 mice at a single time point rather than at different time stages. As such, we did not assess whether ferroptosis occurs prior to or concurrently with neuroinflammation, and it remains unclear whether a feedback loop between these processes exists. Additionally, the relationship between Nrf2/GPX4 and dopaminergic terminal signaling pathways, as well as IKK/IKB/NF-kB signaling, remains to be explored.

\section{Conclusions}

This study demonstrated that FA treatment exerted anti-AD properties via modulation of ferroptosis-mediated neuroinflammation by targeting the activation of the Nrf2/GPX4 axis. Our data suggest that inactivation of the Nrf2/GPX4 axis activates NF- $\mathrm{kB}$ signaling, which further aggravates neuroinflammation (Fig. S12). Our study systematically demonstrates the multitarget protective effects of FA and highlights its therapeutic potential as a promising drug component for the treatment of $\mathrm{AD}$.

\section{Abbreviations}

AD: Alzheimer's disease; $A \beta$ : amyloid- $\beta$; Adcy5: adenylate cyclase 5; AKT: protein kinase B; ALOX5: arachidonate 5-lipoxygenase; BDNF: brain-derived neurotrophic factor; cAMP: cyclic adenosine monophosphate; CREB: cAMP-response element binding protein; DRD1: dopamine receptor D1; DA: dopamine; DMT1: divalent metal-ion transporter-1; ELISA: enzyme-linked immunosorbent assay; FA: forsythoside A; FTH: ferritin heavy chain; FTL: ferritin light chain; FPN: ferroportin; GPX4: glutathione peroxidase 4; GNAL: guanine nucleotide-binding protein $\mathrm{G}($ olf $)$ subunit $\alpha$; GFAP: 
glial fibrillary acidic protein; H\&E: hematoylin and eosin; HO-1: heme oxygenase-1; ІкB: inhibitor of NF-кB; IKK: ІкB kinase; Iba1: ionized calcium binding adapter molecule 1; iNOS: inducible nitric oxide synthase; IL: interleukin; LPS: lipopolysaccharide; MWM: Morris water maze; MMP: mitochondrial membrane potential; MDA: malondialdehyde; MCP-1: monocyte chemoattractant protein-1; NO: nitric oxide; NF-kB: nuclear factor-kB; Nrf2: nuclear factor erythroid 2-related factor 2; NQO1: $\mathrm{NAD}(\mathrm{P}) \mathrm{H}$ quinone dehydrogenase 1; PBS: phosphate buffer solution; PKA: protein kinase A; GSK-3 $\beta$ : glycogen synthase kinase-3 $\beta$; PI3K: phosphatidylinositol-3kinase; ROS: reactive oxygen species; RNAi: RNA interference; RIPA: radio immunoprecipitation assay; siRNA: small interfering RNA; TNF: tumor necrosis factor; TEM: transmission electron microscopy; TGF- $\beta$ : transforming growth factor- $\beta$; TFRC: transferrin receptor; TrkB: tyrosine kinase receptor $\mathrm{B}$; xCT: cystine/glutamate transporter.

\section{Supplementary Material}

Supplementary methods, figures and tables. https://www.ijbs.com/v18p2075s1.pdf

\section{Acknowledgements}

This work was supported by the Jilin Province Science and Technology Development Plan (Medicine and Health Special Project) (20200708037YY and 20200708068YY).

\section{Competing Interests}

The authors have declared that no competing interest exists.

\section{References}

1. Wang CY, Zhang $Q$, Xun Z, Yuan L, Li RN, Li X, et al. Increases of iASPP-Keap1 interaction mediated by syringin enhance synaptic plasticity and rescue cognitive impairments via stabilizing Nrf2 in Alzheimer's models. Redox Biol. 2020; 36: 101672.

2. 2021 Alzheimer's disease facts and figures. Alzheimers Dement. 2021; 17: 327-406.

3. Scheltens P, De Strooper B, Kivipelto M, Holstege H, Chetelat G, Teunissen CE, et al. Alzheimer's disease. Lancet. 2021; 397: 1577-90.

4. Cummings J. New approaches to symptomatic treatments for Alzheimer's disease. Mol Neurodegener. 2021; 16: 2.

5. Dhillon S. Aducanumab: First Approval. Drugs. 2021; 81: 1437-43.

6. Cuddy LK, Prokopenko D, Cunningham EP, Brimberry R, Vassar RJ. $\mathrm{A} \beta$-accelerated neurodegeneration caused by Alzheimer's-associated ACE variant $\mathrm{R} 1279 \mathrm{Q}$ is rescued by angiotensin system inhibition in mice. Sci Transl Med. 2020; 12: eaaz2541.

7. Ayton S, Portbury S, Kalinowski P, Agarwal P, Diouf I, Schneider JA, et al. Regional brain iron associated with deterioration in Alzheimer's disease: A large cohort study and theoretical significance. Alzheimers Dement. 2021; 17: 1244-56.

8. Yan H-f, Zou T, Tuo Q-z, Xu S, Li H, Belaidi AA, et al. Ferroptosis: mechanisms and links with diseases. Signal Transduct Target Ther. 2021; 6: 49.

9. Osama A, Zhang JM, Yao J, Yao XJ, Fang JG. Nrf2: a dark horse in Alzheimer's disease treatment. Ageing Res Rev. 2020; 64: 101206.

10. Yoo S-E, Chen L, Na R, Liu Y, Rios C, Van Remmen H, et al. Gpx4 ablation in adult mice results in a lethal phenotype accompanied by neuronal loss in brain. Free Radic Biol Med. 2012; 52: 1820-7.

11. Onyango IG, Jauregui GV, Carna M, Bennett JP, Stokin GB. Neuroinflammation in Alzheimer's Disease. Biomedicines. 2021; 9: 524
12. Qu Z, Sun JC, Zhang WN, Yu JQ, Zhuang CL. Transcription factor NRF2 as a promising therapeutic target for Alzheimer's disease. Free Radic Biol Med. 2020; 159: 87-102.

13. Li C, Deng XB, Xie XW, Liu Y, Angeli JPF, Lai LH. Activation of Glutathione Peroxidase 4 as a Novel Anti-inflammatory Strategy. Front Pharmacol. 2018; 9: 1120 .

14. Wang ZY, Xia Q, Liu X, Liu WX, Huang WZ, Mei X, et al. Phytochemistry, pharmacology, quality control and future research of Forsythia suspensa (Thunb.) Vahl: A review. J Ethnopharmacol. 2018; 210: 318-39.

15. Wang H-M, Wang L-W, Liu X-M, Li C-L, Xu S-P, Farooq A-D. Neuroprotective effects of forsythiaside on learning and memory deficits in senescenceaccelerated mouse prone (SAMP8) mice. Pharmacol Biochem Behav. 2013; 105: 134-41.

16. Kim JM, Kim S, Kim DH, Lee CH, Park SJ, Jung JW, et al. Neuroprotective effect of forsythiaside against transient cerebral global ischemia in gerbil. Eur J Pharmacol. 2011; 660: 326-33.

17. Chen LQ, Yan Y, Chen TG, Zhang LW, Gao XX, Du CH, et al. Forsythiaside prevents beta-amyloid-induced hippocampal slice injury by upregulating 2-arachidonoylglycerol via cannabinoid receptor 1-dependent NF-kappa B pathway. Neurochem Int. 2019; 125: 57-66.

18. Yan XJ, Chen TG, Zhang LW, Du HZ. Protective effects of Forsythoside A on amyloid beta-induced apoptosis in PC12 cells by downregulating acetylcholinesterase. Eur J Pharmacol. 2017; 810: 141-8.

19. Wang CY, Cai XY, Wang RC, Zhai SY, Zhang YF, Hu WJ, et al Neuroprotective effects of verbascoside against Alzheimer's disease via the relief of endoplasmic reticulum stress in A beta-exposed U251 cells and APP/PS1 mice. J Neuroinflamm. 2020; 17: 309.

20. Wang CY, Hao J, Liu X, Li CL, Yuan XY, Lee RJ, et al. Isoforsythiaside Attenuates Alzheimer's Disease via Regulating Mitochondrial Function Through the PI3K/AKT Pathway. Int J Mol Sci. 2020; 21: 5687.

21. Czapski GA, Cieslik M, Wencel PL, Wojtowicz S, Strosznajder RP, Strosznajder JB. Inhibition of poly (ADP-ribose) polymerase-1 alters expression of mitochondria-related genes in PC12 cells: relevance to mitochondrial homeostasis in neurodegenerative disorders. Biochim Biophys Acta Mol Cell Res. 2018; 1865: 281-8.

22. Lau FC, Joseph JA, McDonald JE, Kalt W. Attenuation of iNOS and COX2 by blueberry polyphenols is mediated through the suppression of NF-kappa B activation. J Funct Foods. 2009; 1: 274-83.

23. Wang Y, Zhao HF, Lin CX, Ren J, Zhang SZ. Forsythiaside A Exhibits Anti-inflammatory effects in LPS-Stimulated BV2 Microglia Cells Through Activation of Nrf2/HO-1 Signaling Pathway. Neurochem Res. 2016; 41: 659-65.

24. Kheirbek MA, Beeler JA, Ishikawa Y, Zhuang X. A cAMP Pathway Underlying Reward Prediction in Associative Learning. J Neurosci. 2008; 28: 11401-8.

25. Herve D. Identification of a specific assembly of the $G$ protein Golf as a critical and regulated module of dopamine and adenosine-activated cAMP pathways in the striatum. Front Neuroanat. 2011; 5: 48

26. Wang D, Peng Y, Xie Y, Zhou B, Sun X, Kang R, et al. Antiferroptotic activity of non-oxidative dopamine. Biochem Biophys Res Commun. 2016; 480: 602-7.

27. Xia QP, Cheng ZY, He L. The modulatory role of dopamine receptors in brain neuroinflammation. Int Immunopharmacol. 2019; 76: 105908.

28. Hao JR, Sun N, Lei L, Li XY, Yao B, Sun K, et al. L-Stepholidine rescues memory deficit and synaptic plasticity in models of Alzheimer's disease via activating dopamine D1 receptor/PKA signaling pathway. Cell Death Dis. 2015; 6: e1965.

29. Khan MM, Xiao JF, Hollingsworth TJ, Patel D, Selley DE, Ring TL, et al. Gnal haploinsufficiency causes genomic instability and increased sensitivity to haloperidol. Exp Neurol. 2019; 318: 61-70.

30. Hong Z-Y, Yu S-S, Wang Z-J, Zhu Y-Z. SCM-198 Ameliorates Cognitive Deficits, Promotes Neuronal Survival and Enhances CREB/BDNF/TrkB Signaling without Affecting A beta Burden in A beta PP/PS1 Mice. Int J Mol Sci. 2015; 16: 18544-63.

31. Yi JM, Zhu JJ, Wu J, Thompson CB, Jiang XJ. Oncogenic activation of PI3K-AKT-mTOR signaling suppresses ferroptosis via SREBP-mediated lipogenesis. Proc Natl Acad Sci U S A. 2020; 117: 31189-97.

32. Ishii T, Warabi E, Mann GE. Circadian control of BDNF-mediated Nrf2 activation in astrocytes protects dopaminergic neurons from ferroptosis. Free Radic Biol Med. 2019; 133: 169-78.

33. Jain AK, Jaiswal AK. GSK-3 beta acts upstream of Fyn kinase in regulation of nuclear export and degradation of NF-E2 related factor 2. J Biol Chem. 2007; 282: $16502-10$

34. Chen J, Wang J, Li C, Ding H, Ye J, Xia Z. Dexmedetomidine reverses MTX-induced neurotoxicity and inflammation in hippocampal HT22 cell lines via NCOA4-mediated ferritinophagy. Aging 2021; 13: 6182-93.

35. Houari S, Picard E, Wurtz T, Vennat E, Roubier N, Wu TD, et al. Disrupted Iron Storage in Dental Fluorosis. J Dent Res. 2019; 98: 994-1001.

36. Hou LY, Huang RX, Sun FQ, Zhang L, Wang QS. NADPH oxidase regulates paraquat and maneb-induced dopaminergic neurodegeneration through ferroptosis. Toxicology. 2019; 417: 64-73.

37. Heneka MT, Carson MJ, El Khoury J, Landreth GE, Brosseron F, Feinstein DL, et al. Neuroinflammation in Alzheimer's disease. Lancet Neurol. 2015; 14: 388-405.

38. Dawson J, Miltz W, Mir AK, Wiessner C. Targeting monocyte chemoattractant protein-1 signalling in disease. Expert opin ther targets. 2003; 7: 35-48. 
39. Chen F, Ghosh A, Lin JR, Zhang CT, Pan YN, Thakur A, et al. 5-lipoxygenase pathway and its downstream cysteinyl leukotrienes as potential therapeutic argets for Alzheimer's disease. Brain Behav Immun. 2020; 88: 844-55.

40. Griciuc A, Federico AN, Natasan J, Forte AM, McGinty D, Nguyen H, et al. Gene therapy for Alzheimer's disease targeting CD33 reduces amyloid beta accumulation and neuroinflammation. Hum Mol Genet. 2020; 29: 2920-35.

41. Yang Y, Luo M, Zhang K, Zhang J, Gao T, O' Connell D, et al. Nedd4 ubiquitylates VDAC2/3 to suppress erastin-induced ferroptosis in melanoma. Nat Commun. 2020; 11: 433.

42. Song X, Wang X, Liu Z, Yu Z. Role of GPX4-mediated ferroptosis in the sensitivity of triple negative breast cancer cells to gefitinib. Front Oncol. 2020: 597434.

43. Battaglia AM, Chirillo R, Aversa I, Sacco A, Costanzo F, Biamonte F. Ferroptosis and Cancer: Mitochondria Meet the "Iron Maiden" Cell Death. Cells. 2020; 9: 1505

44. Wang Q, Bin C, Xue Q, Gao Q, Huang A, Wang K, et al. GSTZ1 sensitizes hepatocellular carcinoma cells to sorafenib-induced ferroptosis via inhibition of NRF2/GPX4 axis. Cell Death Dis. 2021; 12: 426.

45. Bahn G, Park JS, Yun UJ, Lee YJ, Choi Y, Park JS, et al. NRF2/ARE pathway negatively regulates BACE1 expression and ameliorates cognitive deficits in mouse Alzheimer's models. Proc Natl Acad Sci U S A. 2019; 116: 12516-23.

46. Wang $X$, Sun $G$, Feng T, Zhang J, Huang X, Wang T, et al. Sodium oligomannate therapeutically remodels gut microbiota and suppresses gut bacterial amino acids-shaped neuroinflammation to inhibit Alzheimer's disease progression. Cell Res. 2019; 29: 787-803.

47. Bronzuoli MR, Iacomino A, Steardo L, Scuderi C. Targeting neuroinflammation in Alzheimer's disease. J inflamm res. 2016; 9: 199-208.

48. Cavallucci V, D'Amelio M, Cecconi F. A beta Toxicity in Alzheimer's Disease. Mol Neurobiol. 2012; 45: 366-78.

49. Yoon S, Kim YK. The role of immunity and neuroinflammation in genetic predisposition and pathogenesis of Alzheimer's disease. Aims Genet. 2015; 2: 230-49.

50. Storga D, Vrecko K, Birkmayer JG, Reibnegger G. Monoaminergic neurotransmitters, their precursors and metabolites in brains of Alzheimer patients. Neurosci lett. 1996; 203: 29-32.

51. D'Amelio M, Puglisi-Allegra S, Mercuri N. The role of dopaminergic midbrain in Alzheimer's disease: Translating basic science into clinical practice. Pharmacol Res. 2018; 130: 414-9.

52. Nobili A, Latagliata EC, Viscomi MT, Cavallucci V, Cutuli D, Giacovazzo G, et al. Dopamine neuronal loss contributes to memory and reward dysfunction in a model of Alzheimer's disease. Nat Commun. 2017; 8: 14727.

53. Tian J, Guo L, Sui SM, Driskill C, Phensy A, Wang Q, et al. Disrupted hippocampal growth hormone secretagogue receptor 1 alpha interaction with dopamine receptor D1 plays a role in Alzheimer's disease. Sci Transl Med. 2019; 11: eaav6278.

54. Gonzalez GA, Montminy MR. Cyclic AMP stimulates somatostatin gene transcription by phosphorylation of CREB at serine 133. Cell. 1989; 59: 675-80.

55. Suzuki A, Fukushima H, Mukawa T, Toyoda H, Wu L-J, Zhao M-G, et al. Upregulation of CREB-Mediated Transcription Enhances Both Short- and Long-Term Memory. J Neurosci. 2011; 31: 8786-802.

56. Yamamoto-Sasaki M, Ozawa H, Saito T, Rosler M, Riederer P. Impaired phosphorylation of cyclic AMP response element binding protein in the hippocampus of dementia of the Alzheimer type. Brain Res. 1999; 824: 300-3.

57. Yiu AP, Rashid AJ, Josselyn SA. Increasing CREB Function in the CA1 Region of Dorsal Hippocampus Rescues the Spatial Memory Deficits in a Mouse Model of Alzheimer's Disease. Neuropsychopharmacol. 2011; 36: 2169-86.

58. Chen YM, Huang XW, Zhang YW, Rockenstein E, Bu GJ, Golde TE, et al. Alzheimer's beta-Secretase (BACE1) Regulates the cAMP/PKA/CREB Pathway Independently of beta-Amyloid. J Neurosci. 2012; 32: 11390-5.

59. Li C, Sui C, Wang W, Yan J, Deng N, Du X, et al. Baicalin Attenuates OxygenGlucose Deprivation/Reoxygenation-Induced Injury by Modulating the BDNF-TrkB/PI3K/Akt and MAPK/Erk1/2 Signaling Axes in NeuronAstrocyte Cocultures. Front Pharmacol. 2021: 599543.

60. Ates G, Goldberg J, Currais A, Maher P. CMS121, a fatty acid synthase inhibitor, protects against excess lipid peroxidation and inflammation and alleviates cognitive loss in a transgenic mouse model of Alzheimer's disease. Redox Biol. 2020; 36: 101648.

61. El Hout M, Dos Santos L, Hamai A, Mehrpour M. A promising new approach to cancer therapy: Targeting iron metabolism in cancer stem cells. Semin Cancer Biol. 2018; 53: 125-38.

62. Urrutia PJ, Borquez DA, Nunez MT. Inflaming the Brain with Iron. Antioxidants. 2021; 10: 61.

63. Wang J, Song N, Jiang H, Wang J, Xie J. Pro-inflammatory cytokines modulate iron regulatory protein 1 expression and iron transportation through reactive oxygen/nitrogen species production in ventral mesencephalic neurons. Biochim Biophys Acta. 2013; 1832: 618-25.

64. Kwon HS, Koh SH. Neuroinflammation in neurodegenerative disorders: the roles of microglia and astrocytes. Transl Neurodegener. 2020; 9: 42

65. Li CH, Wang J, Fang YQ, Liu Y, Chen T, Sun H, et al. Nafamostat mesilate improves function recovery after stroke by inhibiting neuroinflammation in rats. Brain Behav Immun. 2016; 56: 230-45.

66. Gruol DL. IL-6 regulation of synaptic function in the CNS. Neuropharmacol. 2015; 96: 42-54.
67. Li C, Deng X, Zhang W, Xie X, Conrad M, Liu Y, et al. Novel Allosteric Activators for Ferroptosis Regulator Glutathione Peroxidase 4. J Med Chem. 2019; 62: 266-75.

68. Ma H, Wang $\mathrm{X}$, Zhang W, Li H, Zhao W, Sun J, et al. Melatonin suppresses ferroptosis induced by high glucose via activation of the Nrf2/HO-1 signaling pathway in type 2 diabetic osteoporosis. Oxid med cell longev. 2020; 2020: 9067610.

69. Tao WW, Hu YW, Chen ZY, Dai YX, Hu Y, Qi MM. Magnolol attenuates depressive-like behaviors by polarizing microglia towards the M2 phenotype through the regulation of Nrf2/HO-1/NLRP3 signaling pathway. Phytomedicine. 2021; 91: 153692. 